\title{
I dilemmi del giornalismo: le sfide di internet per il giornalismo professionale e la sostenibilità dei media
}

\section{Eugenia Siapera ${ }^{1}$}

\section{INTRODUZIONE}

Pur essendo discussa l'utilità della nozione di "innovazione di disturbo" (disruptive innovation) (Christensen 1997), si dubita poco del fatto che internet abbia fortemente danneggiato i modi in cui il giornalismo viene prodotto, distribuito e consumato. Non si può sottovalutarne l'impatto, anche se, al momento, non siamo in grado di misurarne gli effetti a lungo termine sulle pratiche, sulle norme e sul ruolo sociale e politico del giornalismo. Gli effetti a breve termine negli ultimi vent'anni circa, ovvero da quando il primo giornale fece la propria comparsa online, sono stati profondamente ambigui. Il presente capitolo passerà in rassegna e analizzerà i modi in cui internet ha "sconvolto" il giornalismo anche per identificare alcune delle sfide emerse o che emergeranno nel prossimo futuro. Il fine ultimo della presente analisi consiste nell'identificare uno spazio all'interno del quale il giornalismo possa ancora svolgere il proprio cruciale ruolo socio-politico. L'analisi suggerisce che, nonostante i molti effetti di disturbo della tecnologia, il dilemma del giornalismo rimane il medesimo: come può esso funzionare in maniera autonoma e indipendente, ma comunque sostenibile e vitale?

Il capitolo è organizzato nel modo seguente. Si inizia con uno sguardo generale ed un confronto dei mass media e dei nuovi cicli della comunicazione e dei social network. Tratterà poi gli sviluppi nei termini dei tre procedimenti maggiori coinvolti in questi cicli, ovvero la produzione, $\mathrm{i}$ contenuti ed il consumo o l'uso. Si concluderà con una sintesi degli sviluppi, delle tendenze e delle sfide principali e con una proposta per la risocializzazione del giornalismo.

1 School of Communications, Dublin City University, eugenia.siapra@dcu.ie. 


\section{IL PROCEDIMENTO DELLA COMUNICAZIONE (MEDIATA)}

Una delle prospettive teoriche più influenti sulla comunicazione non deriva dalla sociologia, bensì dalla matematica: si tratta della teoria delle comunicazioni trasmissive di Claude Shannon (2001 [1948]), la quale definisce la comunicazione un sistema che coinvolge cinque parti. La figura 2.1 contiene una rappresentazione schematica del sistema di comunicazione.

Figura 2.1 - Un diagramma di comunicazione

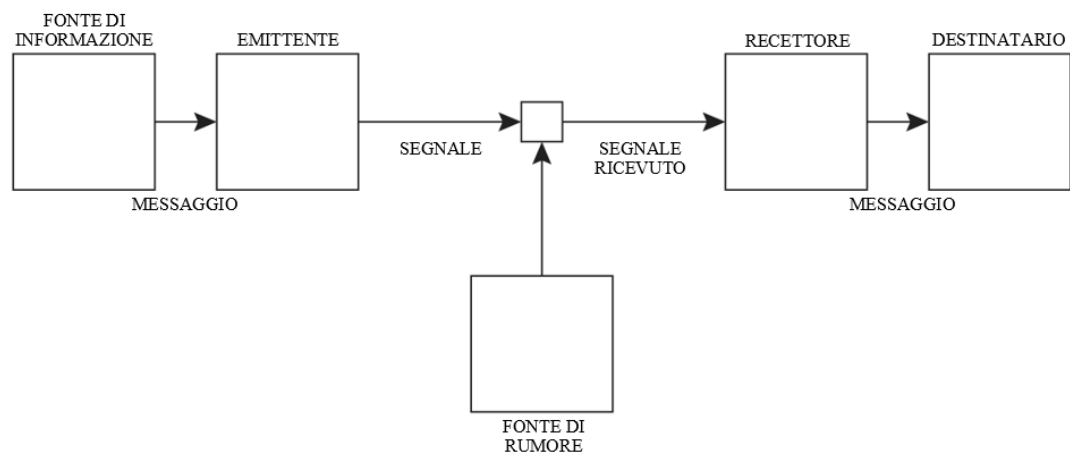

Fonte: Shannon 2001 [1948], p. 2.

Le cinque parti includono (i) una fonte d'informazione, per esempio un giornalista il quale produce un messaggio che viene poi codificato in un segnale pronto per essere trasmesso; (ii) un trasmettitore, per esempio le rotative o la videocamera, che trasforma il messaggio in un segnale adatto ad essere trasmesso, (iii) il canale, per esempio il giornale o l'emittente, che trasmette il segnale; (iv) il ricettore, per esempio gli occhi del lettore o lo studio televisivo, il quale aiuta a leggere o decifrare il messaggio; e (v) il destinatario, ovvero, la persona alla quale il messaggio è in realtà destinato. Questo modello pone l'accento sull'assicurare che non vi siano interferenze e che il messaggio possa giungere a destinazione senza impedimenti. La comunicazione è qui considerata in primo luogo una questione di trasmissioni.

Gli approcci politico-economici alla comunicazione ed ai mass media hanno essenzialmente adottato questo modello lineare di comunicazione e si sono focalizzati sulla proprietà dei mezzi di produzione e di distribuzione dei messaggi mediatici. L'analisi politico-economica dei media classici 
dimostra che i processi di concentrazione della proprietà hanno un impatto significante sulla varietà dei messaggi dei media, limitandoli. Nei loro studi decisivi, Murdock e Golding (1973) hanno identificato tre procedimenti di concentrazione: l'integrazione, la diversificazione e l'internazionalizzazione. L'integrazione fa riferimento alle fusioni ed acquisizioni che hanno portato alla creazione di ampie società mediatiche che non solo detengono diversi organi di stampa, ma anche le unità di distribuzione e di vendita, controllando, di conseguenza, il mercato dei media nella sua quasi totalità. La diversificazione fa invece riferimento al procedimento nel quale un'impresa del settore dei media acquista interessi in altri ambiti, per esempio nelle attività del tempo libero o nella fornitura di informazioni. L'internazionalizzazione coinvolge l'espansione verso diversi mercati nazionali. Per mezzo di questi procedimenti, la produzione di messaggi mediatici è strettamente controllata da pochi grandi conglomerati mediatici. Per il giornalismo ciò si rivela cruciale, in quanto la concentrazione della proprietà comporta una perdita del pluralismo e della diversità nelle voci che circolano nella sfera pubblica.

In ogni caso, l'assunzione dell'approccio politico-economico secondo cui i media detengono l'intero potere e che, assumendo che le interferenze o gli ostacoli sono ridotti o inesistenti, i messaggi possano raggiungere i propri destinatari, è stata messa in discussione. Nel suo dirompente articolo sulla televisione, Stuart Hall (1980) si è focalizzato sulla codifica dei messaggi e sulla successiva decodificazione, ritenendo che si trattasse di "momenti" abbastanza diversi. Hall cercò di ritenere la comunicazione un insieme di momenti distinti, ma collegati, connessi in un circolo. Distingue quattro piani o "momenti" del procedimento: la produzione, la circolazione, il consumo e la riproduzione. La figura 2.2 presenta il modello di Hall in forma schematica. La priorità di questo modello consiste nell'evidenziare il momento di produzione e di recezione quali fasi distinte e nell'indicare fattori come la conoscenza, le norme professionali, il ceto e le ideologie come caratterizzanti della produzione e della recezione. Più che concepire la codificazione come una a questione tecnica di trasformazione di un messaggio in un segnale e gli impedimenti alla decodificazione dovuti a rumori o interferenze nel canale, Hall li considera entrambi prodotti dei diversi fattori di conoscenza e di ceto. Tali differenze possono generare letture negoziate e opposte dei messaggi mediatici. 
Figura 2.2 - Il circuito della comunicazione
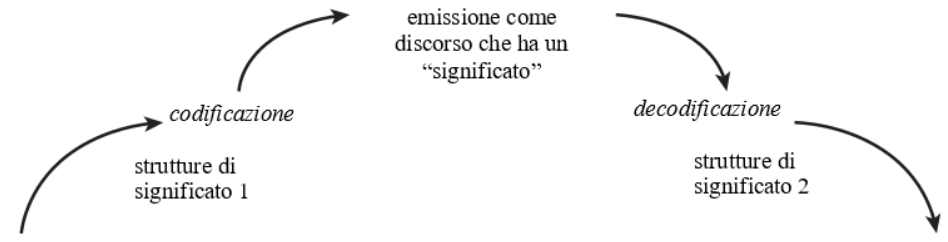

quadro di

conoscenza

quadro di

conoscenza

relazioni di

produzione

relazioni di

produzione

infrastrutture

tecniche

infrastrutture

tecniche

Fonte: Hall 1993 [1980] p. 94.

Nell'articolo in questione, Hall ritiene che il procedimento di comunicazione di massa comprenda sia il produttore che il ricettore del messaggio. In tale procedimento, i produttori sono numericamente inferiori ai ricettori, e la loro codificazione è circoscritta da norme professionali e pratiche, così come dal loro accesso alle infrastrutture tecniche. Mentre gli approcci ai media politico-economici evidenziano il controllo dei mezzi di produzione dei messaggi, Hall si è concentrato maggiormente sui micro-processi, numericamente superiori, di ceti e norme professionali quali contributi ai modi in cui un messaggio viene codificato. In termini di giornalismo, la prospettiva di Hall è cruciale per identificare il ruolo svolto dalle norme professionali e dalle ideologie, così come per dimostrare che i lettori non sono recipienti passivi di informazioni ma parti attive e impegnate. Di conseguenza, un report sui media che contraddica le esperienze e le concezioni personali di una persona andrebbe rigettato. Dall'altro lato, questo modello tende a mettere tra parentesi la questione della circolazione e della distribuzione. Hall include l'infrastruttura tecnica come parte dei due momenti di codificazione e decodificazione, ma dice poco sul coinvolgimento di questi ultimi nel processo comunicativo.

In ogni caso, l'importanza dell'infrastruttura tecnica ed il momento di circolazione/distribuzione sta crescendo se pensiamo a come il procedimento di comunicazione è mutato nell'era dei social media. Uno degli aspetti più dirompenti di internet e dei social media consiste nel fatto che essi consentono a chiunque di produrre contenuti. A circa metà degli anni 
2000, i teorici hanno cominciato a capire che stava accadendo un cambiamento importante quando i prezzi dei computer cominciarono a calare, l'interfaccia stava diventando più user-friendly e le connessioni più veloci $\mathrm{e}$ migliori. Il cambiamento venne descritto in vari modi, ma tutti giungevano alla medesima conclusione: nell'era di internet chiunque è un produttore. Rushkoff (2003), per esempio, parla di un balzo dell'essere autore, Leadbeater e Miller (2004) della rivoluzione "pro-am" (professionale-amatoriale), Rosen (2006) fa riferimento alle "persone precedentemente note come pubblico" e Burns (2006) ai "produsers" (sincrasi di producer e user). Questa raccolta di opere ha definito una realtà in cui le persone non sono più soltanto pubblico, ricettori e decodificatori, bensì loro stessi produttori attivi di contenuti. Questa "democraticizzazione" della produzione mediatica ha drasticamente alterato il modello di comunicazione di massa che si basava su una produzione dei media e su un controllo dei contenuti professionale e sulla distribuzione dello stesso. Come sostenuto da Meikle, i siti web prodotti dagli utenti, come i siti di Indymedia, "pongono l'accento sulla produzione più che sul consumo dei testi mediatici” (2002: 87). Per il giornalismo l'importanza di questo cambiamento non può essere sopravvalutata: il quasi-monopolio di cui godevano i giornalisti professionisti ed i comunicatori è terminato e la posizione relativamente privilegiata dei giornalisti è andata persa.

Sin dalle prime apparizioni della comunicazione su internet e nei social media, vi sono stati cambiamenti significativi che hanno portato ad un ecosistema mediatico in generale diverso all'interno del quale il giornalismo opera. La rapidissima ascesa dei social network quali Facebook e Twitter, la diffusione e la popolarità dei dispositivi mobili quali tablet e smartphone e la crescita della globalizzazione e delle interdipendenze dei mercati e delle società hanno colpito non solo la produzione, ma anche la distribuzione, la forma e lo scopo del giornalismo. I prossimi paragrafi esamineranno questi processi nel dettaglio, elencando una serie di cambiamenti e di sfide per il giornalismo.

\section{UN ECOSISTEMA CHE CAMBIA}

Sulla scia di Hall, questo paragrafo considera i cambiamenti dell'ecosistema mediatico in termini di produzione, distribuzione, contenuti e forma, utenti e usi. Partendo dal modello di Hall, questa sezione non privilegia la produzione o la recezione né ritiene questi momenti equivalenti. Si dirà invece che il processo di distribuzione emerge in modo privilegiato in considerazione del modo in cui opera l'intero ecosistema. Ciò indirizza, a sua 
volta, gli sviluppi in tutti gli altri processi determinando risultati molto ambigui per il giornalismo.

\subsection{Produrre il giornalismo ${ }^{2}$}

I cambiamenti possono qui essere affrontati in termini di fonti di guadagno del giornalismo ed in termini di lavoro e di pratiche del giornalismo. I cambiamenti nell'ecosistema mediatico hanno coinvolto l'intero modello imprenditoriale del giornalismo concepito come "architettura per la produzione, il servizio e i flussi di informazioni, inclusa una descrizione dei vari attori imprenditoriali e dei loro ruoli; ed una descrizione delle fonti di guadagno" (Timmers 1998:2). Mentre risulta difficile separare la componente economica del giornalismo da quella sociale, questa definizione fornisce un'euristica utile sulla base della quale possiamo definire le differenze tra il modello precoce di mass-media del giornalismo e l'attuale modello digitale e sociale. Il presente paragrafo presume una prospettiva storica, volgendo l'attenzione verso l'evoluzione del giornalismo e la sua transizione da modello industriale a modello digitale.

\subsubsection{L'evoluzione del giornalismo industriale}

Nel suo giudizio sulla sfera pubblica, Habermas (1991) traccia tre livelli nello sviluppo del giornalismo: dalla vendita di notizie passando per la vendita di opinioni fino alla vendita di spazi pubblicitari. Nei suoi primi livelli, il giornalismo si esplicava in un'attività di commercio di notizie. Secondo l'analisi di Bücher (1926, citata in Habermas 1991), Habermas afferma che il giornalismo iniziò come sistema di corrispondenze private, le quale vennero poi raccolte e unite da parte di un editore al fine di venderle ai lettori interessati. In questa fase il giornalismo era essenzialmente un commercio di notizie "pure". Questo tipo di giornalismo conobbe poi il giornalismo formale degli "intellettuali", il quale forniva opinioni e critiche, portando ad un nuovo genere di giornalismo in cui le notizie intese come semplici informazioni divennero secondarie rispetto alle concezioni editoriali ed alle opinioni. Questo tipo di giornalismo, scrive Habermas, non ebbe successo commerciale e sopravvisse soltanto essendo "il passatempo dell'aristocrazia monetaria" nel Regno Unito e il risultato di inizia-

2 Il termine "giornalismo industriale" è stato coniato da Anderson et al. (2012). 
tive personali e dell'impegno di persone acculturate sul continente (Habermas d1991: 182). A questo livello, l'operazione commerciale della stampa non era tanto importante quanto l'affermazione della funzione di critica del pubblico. Come evidenziato da Bücher, il giornalismo mutò qui dall'essere "un mercante di notizie all'essere un fornitore dell'opinione pubblica” (Bücher (1926), citato in Habermas 1991: 182). In ogni modo, una serie di sviluppi a circa metà dell'Ottocento, inclusi la revoca delle imposte riscosse sulla stampa, l'aumento dei costi di produzione ed i bassi prezzi di copertina (Curran e Seaton 2003), portarono alla necessità della creazione di grandi imprese del settore mediatico dotate di risorse finanziarie maggiori. Curran e Seaton hanno rilevato che, nel 1830, servirono soltanto 690 sterline per fondare Northern Star, un settimanale a diffusione nazionale nel Regno Unito, e che tale spesa poté essere recuperata dalla vendita di circa 6.200 copie, un numero raggiunto nel primo mese di pubblicazione. Nel 1918, il Sunday Express richiese un investimento di oltre 2 milioni di sterline, il quale venne recuperato solo dalla vendita di circa 250.000 pezzi. La vendita non bastò più. I giornali si rivolsero alla pubblicità, la vendita di spazi pubblicitari non divenne più sussidiaria alla vendita delle copie stampate, bensì costituì la prima fonte di guadagno.

Tale affidamento alla pubblicità aveva avuto un impatto significante sui modi in cui veniva organizzato e prodotto il giornalismo. La principale strategia di mercato non era più costituita dalla vendita di notizie ai lettori, bensì nel fornire pubblico agli inserzionisti. Da ciò nacquero forti pressioni sui giornalisti affinché richiamassero un pubblico maggiore o più specifico a seconda delle esigenze degli inserzionisti. Secondo Dallas Smythe (1981: 241) "per i giornali e le riviste, gli inserzionisti pagano la maggior parte dei costi - tipicamente dal 70 al $90 \%$. Gli abbonamenti e le vendite nelle edicole coprono approssimamente solo i costi di spedizione dei giornali e delle riviste." Secondo la tesi principale di Dallas Smythe (1981) il pubblico rappresenta la merce che i media vendono successivamente agli inserzionisti. In aggiunta, per causa del controllo monopolistico od oligopolistico del settore mediatico, i media sono poi capaci di stabilire le loro tariffe a proprio vantaggio; per esempio, i pubblichi o lettori nazionali portano prezzi maggiori rispetto ai lettori locali o ai lettori per gli annunci economici.

Posto che il pubblico rappresentava il prodotto principale a disposizione del giornalismo per la vendita di spazi pubblicitari, il principale obiettivo per la sopravvivenza del giornalismo consiste nella ricerca di pubblico o di lettori. I giornali si specializzarono in termini di tipologia di lettori che cercarono di vendere agli inserzionisti. Per esempio, Curran e Seaton (2003) mostrano che, negli anni 30, il quotidiano britannico The Daily He- 
rald vendeva agli inserzionisti lettori appartenenti alla classe dei lavoratori, mentre, nel 1938, The Daily Mirror "lanciò una campagna pubblicitaria rivolta agli inserzionisti vantandosi dei propri lettori altolocati" (Curran e Seaton 2003: 51). Quando il Mirror cercò di reinventarsi come giornale dei lavoratori, ridusse della metà la quantità di notizie dedicate ai temi politici, economici e sociali sostituendoli con articoli che affrontavano tematiche quali il crimine, il sesso e tematiche adatte a richiamare un pubblico femminile e giovane.

Mentre l'esempio del Mirror mette in luce il modo in cui gli editori si concentravano su lettori specifici al fine di farsi notare da inserzionisti specifici, altre tecniche sono onnicomprensive (c.d. bundling), ovvero il giornalismo unisce contenuti diversi al fine di rivolgersi ad un pubblico più ampio con un prodotto singolo (van der Wurff 2012). Tale tecnica rappresenta un modo più efficace per rivolgersi a un pubblico più ampio $\mathrm{e}$, di conseguenza, per ottenere introiti maggiori da una varietà di fonti. Un esempio di bundling consiste nell'inserzione di supplementi nei giornali di oggi con le pagine dedicate all'economia color rosa, inseriti dedicati allo sport o alle compravendite immobiliari e le riviste. In questo modo, essi si rivolgono ad una platea più ampia, assicurandosi gli introiti da vari tipi di inserzionisti. Il bundling, abbinatto alla capacità di utilizzare il giornalismo per vendere sia al pubblico che il pubblico agli inserzionisti, consentì agli editori di raggiungere economie di scala e, di conseguenza, di rafforzare e di consolidare la propria posizione aumentando le concentrazioni, le integrazioni verticali e orizzontali ed elevare gli ostacoli per i nuovi arrivati.

Questo monopolio o oligopolio dei media ha portato al modello mediatico dominante caratterizzato da un ampio mercato controllato essenzialmente da una manciata di conglomerati di dimensioni molto grandi. Nella loro economia politica dei media, Golding e Murdock (1991) propongono quattro processi storici tra loro collegati che possono aiutare a spiegare la produzione del giornalismo, ovvero: la crescita del settore dei media, l'ascesa di società mediatiche, la commodificazione ed il cambiamento del ruolo della regolamentazione governativa. Quando il settore dei media si è industrializzato, ha poi consolidato le posizioni dei propri giganti e si è messo al riparo dalle crisi di mercato. Tutto ciò venne raggiunto tramite fusioni e acquisizioni, ovvero tramite un processo di integrazione. Tali integrazioni possono essere verticali, quando un'azienda del settore mediatico acquisisce altre aziende a livelli diversi, oppure orizzontali, quando un'azienda del settore mediatico rileva altre aziende che si trovano al medesimo livello. L'integrazione orizzontale avviene, per esempio, quando News Corp acquisisce una serie di etichette mediatiche, quali The Times, Wall Street Journal, Fox News Network, BSkyB, HarperCollins, ecc., men- 
tre l'integrazione verticale avviene quando l'acquirente è una holding del settore della produzione e distribuzione mediatica. Questa specie di integrazione verticale e orizzontale globale è stata resa possibile a causa dei cambiamenti nella politica mediatica, e nel dettaglio tramite il modello di crescente deregolamentazione di stampo neoliberale.

Il lavoro giornalistico assomigliava, secondo il modello tradizionale, "industriale" di giornalismo, ad altri tipi di lavoro presenti nelle società industrializzate. Nello specifico, i giornalisti operavano come lavoratori stipendiati ed il lavoro giornalistico era strutturato in maniera gerarchica, frammentato e diviso in compiti separati con controlli incorporati. Applicando il modello dei processi lavorativi al giornalismo, Örnebring (2010) fa riferimento alla graduale introduzione di un modello di organizzazione del lavoro giornalistico gerarchico ed alla crescente differenziazione e specializzazione all'interno della professione. Quello precedente derivava dalla graduale separazione tra "l'ideazione (pianificazione dei contenuti, decisione di cosa stampare, ricerca del materiale e assunzione dei redattori) e l'esecuzione (la redazione in sé e la ricerca delle informazioni)" del giornalismo (Örnebring 2010: 61-62). Ciò corrisponde essenzialmente al modo in cui il giornalismo, inteso come la ricerca di notizie, si è scisso dalla redazione, dalla stampa e dalla divulgazione delle notizie. Nel giornalismo degli inizi, ritiene Örnebring, tutti questi processi vennero svolti dalla medesima persone. Come evidenziato da Schudson (1978: 65), fino al Novecento i giornali erano principalmente "marchi unipersonali"; solo quando divennero un'attività commerciale, come visto precedentemente, che il lavoro del giornalismo mutò e l'ideazione e l'esecuzione del giornalismo divennero processi separati rigidamente delimitati. L'ideazione divenne compito del management e l'esecuzione compito del giornalista in quanto dipendente. Emerse una gerarchia relativamente rigida, al cui vertice si posizionano gli editori, seguiti dai direttori, giornalisti, redattori, ecc. (Örnebring 2010). Malgrado tale struttura gerarchica, non era raro che i redattori si schierassero dalla parte dei giornalisti piuttosto che da quella degli editori nelle controversie, in quanto essi erano avanzati soprattutto dal livello di giornalista e la loro etica e la loro identità professionale erano condivise con quelle dei giornalisti semplici.

In secundis, la separazione tra l'ideazione e l'esecuzione del giornalismo fu accompagnata da una crescente differenziazione dei vari aspetti del lavoro del giornalismo. I "marchi unipersonali" divennero sempre più differenziati e coloro che lavoravano su un aspetto del giornalismo, per esempio la stampa, non avevano nessuna idea dell'attività di inchiesta giornalistica. Ad un certo punto, i reporter erano altamente specializzati nelle tecniche di ricerca di informazioni, ma non nella scrittura, compito dei correttori 
(sub-editors) negli uffici. Negli anni più recenti, la differenziazione ha assunto la forma di "ritmo", per mezzo del quale un giornalista si specializzerebbe in una tipologia di notizie, per esempio la cronaca politica, oppure giudiziaria o sportiva. Inoltre, la comparsa di "staff di supporto" quali fotoreporter o illustratori, evidenzia tale differenziazione all'interno della professione (Örnebring 2010). Tale differenziazione ha stabilito le priorità del giornalismo, in altre parole la raccolta e l'unione di notizie così come lo storytelling, mentre coloro che possiedono competenze tecniche (fotografi, addetti al suono, cameramen, ecc.) vengono considerati ausiliari.

Riassumendo il modello fino a poco tempo fa dominante della produzione giornalistica dalla prospettiva del modello commerciale, possiamo vedere che ciò corrisponde alla produzione industriale di beni, con alcune peculiarità. La prima consiste nel fatto che il modello economico dei media "industriale" faceva leva su due mercati: quello degli inserzionisti e quello dei lettori. La seconda nel fatto che la crescita del settore mediatico ha dato adito all'avanzamento di strutture oligopolistiche con alti tassi di concentrazione ed integrazione. Terzo, la divisione del lavoro nel settore dei media era organizzata sulla base di uno schema gerarchico, con la divisione tra i manager e i lavoratori, dando comunque priorità al giornalismo (ovvero la ricerca di notizie e lo storytelling), almeno finché i dirigenti del quotidiano erano loro stessi giornalisti. Il valore aggiunto di tale modello deriva dalla capacità di rivolgersi sia agli inserzionisti che ai lettori, rendendolo non solo sostenibile, ma anche piuttosto redditizio. Nonostante le varie critiche mosse nei confronti di tale modelli, specialmente quelle basate sulla commodificazione delle notizie (McManus 1994) e sulla commodificazione del pubblico (Dallas Smythe 1981), sulla mutazione del giornalismo in pubbliche relazioni (Habermas 1991) e sull'industria mediatica oligopolistica (Golding e Murdock 1973), tale modello è riuscito a mantenere un determinato livello di produzione giornalistica per oltre un secolo.

\subsubsection{L'interferenza del digitale della produzione}

Questo modello di produzione e circolazione giornalistica venne fermato dall'ascesa di internet. Il fenomeno si è intensificato con la proliferazione dei social media. Tale interferenza ha colpito la produzione giornalistica a livello dei ricavi ed a livello del lavoro giornalistico. Per certi versi, tali cambiamenti seguono di pari passo i mutamenti sociali più ampi legati alla svolta verso un modello neoliberale di organizzazione capitalistica e non vanno considerati un effetto diretto delle nuove tecnologie. Allo stesso tempo, come dimostrato da Örnebring (2010), le nuove tecnologie hanno 
sempre disturbato il giornalismo, nonostante esso sia stato in grado di recepire tali mutamenti. È comunque importante dimostrare che, quando internet fece la propria comparsa, il giornalismo aveva già attraversato una serie di cambiamenti nell'organizzazione e nella produzione, cambiamenti legati alla digitalizzazione ed al crescente uso dei computer sul posto di lavoro. Inoltre, tali cambiamenti avvennero in parallelo rispetto ad ulteriori importanti cambiamenti nell'organizzazione del lavoro, ovvero l'indebolimento dei sindacati ed il crescente managerialismo.

Forse il cambio più paradigmatico è stato quello affrontato da News International (il ramo di News Corp attivo nel Regno Unito) in seguito alla decisione di spostare la produzione a Wapping negli anni 80. Lo spostamento della produzione dalla storica Fleet Street a Wapping nel quartiere Docklands a Londra venne interpretato come mossa contro il sindacato dei tipografi e dei giornalisti e, in secundis, seguito dall'introduzione dei computer nella produzione del giornalismo. Ciò avvenne con lo scopo dichiarato di aumentare gli investimenti e la redditività (Majoribanks 2000). Lo spostamento fu controverso e segnato da confrontazioni, con una rigida gestione sostenuta dall'allora Primo Ministro Margaret Thatcher. Nel proprio rapporto sul trasferimento Majoribanks (2000) spiega i dettagli di come la gestione di News International abbia usato, all'interno del contesto di un forte sostegno da parte del Governo e dell'apparato statale, l'innovazione tecnologica per rimuovere i sindacati ed i lavoratori manuali dalla produzione del giornalismo, licenziando in totale circa 5.500 lavoratori. La nuova forza lavoro sarebbe stata formata utilizzando i sistemi informatici specializzati di News International. L'esempio di News International venne presto seguito da altri giornali nel Regno Unito e nel resto del mondo, seppure in misura diversa ed in modo meno acrimonioso.

Tali importanti mutamenti, ovvero lo smantellamento effettivo dei sindacati, l'ascesa del managerialismo e la computerizzazione del lavoro giornalistico, si erano già verificati quando, circa 15 anni dopo, la comparsa di internet causò una nuova serie di sfide rivolte non solo ai giornalisti stessi e ai loro metodi, ma ad ampi gruppi quali News International. Quando i giornali si spostarono on-line agli albori del World Wide Web, il loro scopo era, di nuovo, quello di aumentare la propria profittabilità tramite le nuove tecnologie. Derek Bishton, il caporedattore del The Daily Telegraph ed il responsabile del lancio dell'Electronic Telegraph, il primo quotidiano online del Regno Unito, ricorda come l'allora proprietario del The Telegraph, Conrad Black, gli disse: "Dovrai lavorare molto duramente per convincermi di investire in qualcosa che offriamo gratuitamente" (Bishton 2001: non numerato). Ma il dado era tratto: dopo il The Telegraph, altri giornali fecero lo stesso, pubblicando gratuitamente on-line i propri contenuti stam- 
pa. Nonostante fosse ancora interessata nelle potenzialità commerciale delle nuove tecnologie, l'evoluzione del giornalismo on-line sortì, di pari passo con l'evoluzione di internet, l'effetto opposto, ovvero lo smantellamento del modello commerciale del giornalismo. Ciò avvenne in due modi diversi: innanzitutto a causa della gratuità dei contenuti on-line e, in secondo luogo, a causa della frammentazione del pubblico di internet e la conseguente perdita della massa di lettori da vendere agli inserzionisti. Il giornalismo on-line non può quindi fare affidamento al duplice mercato degli inserzionisti e dei lettori.

\subsubsection{L'accesso a pagamento}

Nello specifico, il primo approccio verso le notizie on-line prese la forma della pubblicazione di contenuti stampa su internet. Mentre negli anni seguenti ciò venne accompagnato da articoli e commenti dedicati esclusivamente alla sezione on-line, e incrementando i livelli di interattività, l'idea di fondo di postare gratuitamente le notizie rimase immutata, nonostante gli sforzi verso l'inserimento di servizi a pagamento o abbonamenti. Arrese (2015) ha delineato quattro fasi del modo in cui il giornalismo on-line ha cercato di monetizzare il web: inizialmente, nei primi anni (1994-2000), la fase pionieristica e sperimentale, in cui i vari giornali on-line sperimentarono modelli diversi, incluso un servizio per soli abbonati. In ogni modo, ciò non funzionò, in quanto tali giornali non furono in grado di attirare lettori, e tale fase si concluse con un passaggio quasi univoco verso i contenuti gratuiti, in quanto i giornali on-line speravano che ciò sarebbe bastato per attrarre un pubblico ampio e, di conseguenza, gli inserzionisti. Il The Wall Street Journal costituì l'unica eccezione, il quale aveva adottato una strategia di servizi a pagamento e la mantenne. Nella seconda fase (2001-2007), definita da Arrese la "frenesia degli esperimenti non riusciti", i giornali online tentarono e sperimentarono una serie di modelli, per esempio i micropagamenti, i contenuti premium per gli abbonati e la vendita di versioni in pdf stampabili, ma nessuno di questi riscontrò un successo durevole.

La terza fase (2008-2010) venne capitanata dalla conversione di Rupert Murdoch da discepolo dei contenuti gratuiti a difensore di quelli a pagamento. Quando News Corp iniziò a percepire il dolore del calo delle vendite e degli introiti pubblicitari, in quanto sempre più inserzionisti si rivolsero a Google e la crisi finanziaria del 2008 aveva reso più difficili le condizioni anche alle grandi società, Murdoch iniziò a spingere per l'introduzione di un modello di contenuti a pagamento per la maggior parte delle pubblicazioni on-line di News Corp, inclusi i tabloid popolari quali The Sun. Il 
mutamento del modello commerciale venne sintetizzato con parole molto chiare da parte di Murdoch stesso in un editoriale uscito sul The Wall Street Journal: "I contenuti di qualità non sono gratuiti. In futuro, il buon giornalismo dipenderà dalla capacità di un'impresa mediatica di attrarre i clienti offrendo notizie ed informazioni per le quali essi sono disposti a pagare. Il vecchio modello commerciale fondato essenzialmente sulla pubblicità è morto" (Murdoch 2009, A21, citato in Arrese 2015: 9). In altre parole, il giornalismo sarebbe ora a servizio di un solo padrone, i lettori, offrendo loro contenuti di qualità per i quali essi sarebbero disposti a pagare. Una serie di pubblicazioni on-line fece la stessa cosa, ovvero The Economist, l'editore tedesco Springer e i suoi principali giornali Die Welt e Bild, i quotidiani francesi Le Monde e Libération, e molti altri che avevano stabilito che avrebbero cominciato a rendere a pagamento i contenuti (Arrese 2015). Emersero due principali piani di addebito, il modello "freemium" e le versioni misurate: il primo si riferisce alla pratica di offrire alcuni contenuti a titolo gratuito e richiedere l'iscrizione per ulteriori accessi, ed il secondo a quella di offrire un determinato numero di articoli gratis per poi vendere gli ulteriori accessi. Nonostante tali richiami, alcuni dei maggiori editori non adottarono la nuova strategia, p.es. The Guardian e The Huffington Post, i quali mantennero il loro modello di contenuti gratuiti.

La fase finale e attuale, sostiene Arrese (2015), venne inaugurata con la decisione del The New York Times di applicare un sistema di pagamenti "impermeabile", sulla scia delle versioni misurate. Ciò divenne un punto critico per la (ri)adozione del modello di notizie a pagamento da parte di un numero consistente di editori in tutto il mondo. La realtà della sopravvivenza ha spinto molti player importanti a cercare una chiara fonte di guadagni ed i modelli di pagamento, "impermeabili" o meno, possono rivelarsi necessari. Allo stesso tempo, Arrese evidenzia che vi sono ancora molte sperimentazioni, con gli editori che cercano di bilanciare gli introiti provenienti dagli inserzionisti con quelli provenienti dai lettori. Altri giornali, per esempio il danese De Correspondent, il quale ha superato il record del crowdfunding nel giornalismo raccogliendo un milione di euro da 15.000 sottoscrittori in otto giorni, operano utilizzando un modello di sole sottoscrizioni, mentre un'altra start-up danese, Blendle, opera come piattaforma che unisce contenuti per i quali i lettori pagano i singoli articoli. Sia $D e$ Correspondent che Blendle si sono rilevati economicamente sostenibili fino ad ora.

In ogni modo, vi sono una serie di fattori che continuano a fare pressione sull'applicabilità dei modelli a pagamento nel giornalismo on-line: in prmis, vi sono tutt'ora importanti ed influenti produttori di notizie che offrono contenuti a titolo gratuito e che difficilmente cambieranno strategia, 
p.es. le emittenti pubbliche quali la BBC, oppure editori devoti al giornalismo libero, p.es. The Guardian. Altre categorie includono, in questa sede, $\mathrm{i}$ nuovi arrivati che vogliono costruirsi la loro fascia di lettori offrendo contenuti gratuiti. La conclusione è che, in questo momento, esistono tutt'ora molti contenuti gratuiti e che i lettori non potrebbero essere ancora disposti a pagare per i contenuti. Questa riluttanza potrebbe anche essere un fattore culturale. Per esempio, nei Paesi del Sud Europa, ovvero l'Italia, la Grecia, la Spagna e il Portogallo, i sistemi a pagamento hanno riscontrato un successo ancora minore in confronto al Nord Europa ed agli Stati Uniti, evidenziando che si tratta di ragioni culturali. Il ruolo degli utenti nella produzione, nel consumo e, di conseguenza, nei mutamenti del giornalismo non va sottostimato. Gli utenti e le loro abitudini verranno trattate nello specifico nei paragrafi che seguono. Un terzo fattore che fa dubitare del successo dei sistemi a pagamenti è il ruolo sempre più importante dei social media nella divulgazione di notizie. Le persone fanno infatti sempre più affidamento ai social media quali Facebook per reperire notizie e per godere della cultura della condivisione dei social media, non intravedendo, di conseguenza, nessun valore nel pagare per abbonarsi ad un singolo organo di stampa i cui contenuti non possono essere condivisi. Inoltre, vi sono svariati modi o attacchi informatici che consentono agli utenti di bypassare i sistemi a pagamento accedendo ai contenuti senza dover pagare. Per esempio, con l'uso di server di tipologia proxy si possono bypassare i modelli "misurati" ("metered"), mentre tramite una ricerca su Google sulla base del titolo di, per esempio, un articolo del Wall Street Journal, si può accedere all'intero contenuto dello stesso (vedi Smith 2015). Infine, gli editori che fanno affidamento sulla pubblicità devono affrontare il crescente utilizzo dei c.d. "ad blocker", utilizzati per bloccare le pubblicità sui browser di coloro che installano tali programmi. Ciò rende gli inserzionisti sempre più restii a pagare per i c.d. "banner". Si tratta di sfide importanti che il giornalismo deve affrontare nella propria ricerca di un modello commerciale sostenibile e remunerativo.

\subsubsection{Gli sforzi del giomalismo nell'eradigitale}

La digital disruption, in ogni modo, non si è verificata soltanto a livello di incassi. Ha anche colpito significativamente il lavoro giornalistico. Sono stati infatti coinvolti in maniera più diretta tre aspetti principali del lavoro giornalistico: in primis, il modo in cui è organizzato il lavoro giornalistico è cambiato radicalmente a causa della compressione del tempo e della produzione di un giornalismo a più livelli. In secondo luogo, l'utilizzo delle 
nuove tecnologie nel giornalismo ha coinvolto lo strumentario necessario per svolgere il giornalismo. E, terzo, il rapporto tra la gestione ed i giornalisti si è fortemente alterato, con i giornalisti che lavorano come free-lancers sulla base di contratti precari a tempo determinato.

Esaminando ulteriormente tali sviluppi, l'ascesa di un circolo di informazione attivo 24 ore al giorno 7 giorni su 7 è stata, di fatto, accompagnata dalla televisione satellitare e da canali tv dedicati esclusivamente all'informazioni quali la CNN (vedi Cushion e Lewis 2010), ma internet ha intensificato tale fenomeno. Mentre la carta stampata richiede che i giornalisti raccolgano e riportino le notizie una o due volte al giorno, e mentre i notiziari televisivi facevano ugualmente leva su una raccolta di notizie effettuata una o due volte al giorno, in tempo per il telegiornale dell'ora di pranzo e della sera, internet richiede, al contrario, un aggiornamento costante. In altre parole, per la maggior parte, i giornalisti che lavoravano per i giornali stampati o per le emittenti televisive operavano sulla base di una "deadli$n e$ ". Su internet, invece, non vi è alcuna "deadline": vi è invece la necessità di aggiornamenti costanti, di notizie pubblicate in tempo reale e spesso l'esigenza di aggiornare una notizia seppure non vi siano stati nuovi sviluppi al fine di catturare l'attenzione dei lettori. Paulussen (2012) riferisce che i giornalisti on-line ritengono che il loro lavoro richieda una fornitura di notizie costante e in tempo reale, mentre Schmitz Weiss e de Macedo Higgings Joyce (2009: 599) osservano che la produzione di notizie avviene ora "attraverso una dimensione temporale compressa". Similmente, Örnebring (2010: 65) tratta il tema della velocità collegato all'introduzione delle nuove tecnologie nelle redazioni, sostenendo che la "funzione prima di qualsiasi nuova tecnologia consiste nel velocizzare il processo informativo". Inoltre, a sempre più giornalisti viene chiesto di produrre le notizie non soltanto per internet, ma anche per la carta stampata e spesso anche per le trasmissioni (televisive o radio). Ciò ha determinato un aumento considerevole del carico di lavoro che grava su di essi - Lee-Wright, Philips e Witchge (2011) riportano che un turno di 15 ore non è inusuale - imponendo ai giornalisti tempi ancora più stretti.

Le nuove tecnologie hanno anche colpito lo strumentario di cui sono in possesso i giornalisti. Sembra esservi un ampio consenso sulla necessita di possedere varie competenze e di acquisire conoscenze sulla tecnologia per i giornalisti (Ursell 2001; Garcia-Avile et al. 2004; Örnebring 2010; Paulussen 2012). Senza ombra di dubbio lo strumentario richiesto oggi ai giornalisti è molto diverso da quello richiesto 25 anni fa; bisogna, tuttavia, sottolineare che le competenze tecnologiche vengono considerate aggiuntive rispetto alle competenze tradizionali di cui dovevano disporre i giornalisti "industriali". Abilità quali la creazione e la pubblicazione di foto, la reda- 
zione di filmati tramite telefono cellulare, scrivere blog, guidare e commentare storie, come affermato da Jana Singer (2010: 105), "si aggiungono alle competenze richieste alle passate generazioni di giornalisti”. Si è affermata la figura del c.d. "giornalista-zaino" ("backpack journalist"), un professionista multidisciplinare in grado di produrre il giornalismo tramite lo strumentario nel proprio zaino. Riguardo a ciò, le nuove tecnologie sembrano coprire le divisioni e differenziazioni interne nel giornalismo in quanto il "sostegno" ed il supporto "cardine" si fondono.

D'altro canto, come vedremo nel capitolo 3.2 dedicato alla forma ed ai contenuti, le nuove forme di giornalismo quali il giornalismo dei dati, il giornalismo immersivo ed il giornalismo mobile, richiedono conoscenze speciali e la comprensione di tecnologie e applicazioni specifiche. Forse più delle altre forme, il giornalismo dei dati richiede una conoscenza approfondita dell'analisi dei dati, così come nuove tecniche di presentazione, quali tecniche di visualizzazione, mappe dati o infografiche. Sembra quindi che almeno in questo ambito vi sia in gioco una dinamica diversa che riporta alla specializzazione ed alla "separazione" del giornalismo in subspecialismi.

Parallelamente alle abilità tecniche richieste, vi è un'altra serie di competenze rivelatasi importante per il giornalismo. Si tratta delle abilità sociali di gestione ed interazione con i lettori. È la componente affettiva del giornalismo la quale è, specialmente nei social media, vitale e forse più importante del riporto di notizie. Il lavoro affettivo del giornalismo riguarda la costruzione e la gestione di reti di lettori e la formazione di legami tra i giornalisti e il pubblico/la rete che essi servono (Siapera e Illiadi 2015). Lewis et al. (2014) lo definiscono giornalismo reciproco e lo ritengono esclusivamente positivo, in quanto contribuisce alla creazione di fiducia, connessioni e capitale sociale. Siapera e Iliadi (2015) ritengono, in ogni modo, tale elemento del lavoro giornalistico profondamente ambiguo nel senso che include una tensione irrisolta tra la commodificazione dei rapporti sociali ed il potenziale di creare nuovi legami sociali e nuove soggettività giornalistiche. Tale questione verrà affrontata in maniera più dettagliata nel capitolo 3.4.3.

Oltre al tempo coinvolto ed alla serie di competenze, l'interferenza digitale ha poi riorganizzato la relazione tra la gestione ed i giornalisti. La caratteristica principale di tale riorganizzazione consiste nell'ascesa di contratti precari e del giornalismo free-lance. Deuze (2009) è arrivato a definire gli editori "quelli precedentemente noti come datori di lavoro", parafrasando la nozione di pubblico "precedentemente noto come spettatori" di Jay Rosen (2006). Vi sono, in breve, vari indicatori che dimostrano che il giornalismo ha seguito tendenze più ampie nel lavoro e nell'occupazione che 
hanno determinato uno spostamento da occupazioni permanenti a lungo termine con un datore di lavoro stabile verso forme di lavoro flessibili a breve termine e basate su singoli progetti. Come evidenziato da Castell (2000), tale cambiamento riguarda l'individualizzazione del rapporto tra lavoro e capitale cosicché, in termini giornalistici, esso non è più governato da sindacati o contratti di lavoro collettivi, bensì da accordi individuali tra giornalisti ed editori/imprese del settore dei media. Questo c.d. "lavoro atipico" inteso come free-lance, lavoro sulla base di contratti a tempo determinato (Waters, Warren e Dobbie 2006; Deuze e Fortunati 2010) sta diventando sempre più tipico per i giornalisti.

L'impatto esatto di tutto ciò sulla prassi giornalistica è difficile da misurare. Si può, tuttavia, tranquillamente affermare che questi sviluppi sono ambigui. Da un lato, il free-lancing offre maggiore libertà e autonomia ai giornalisti, di conseguenza arricchendo il loro lavoro di soddisfazione e creatività (Massey ed Elmore 2011). Dall'altro lato, un recente studio sui giornalisti nel Sud-Est Europa e in Italia richiama la maggiore vulnerabilità dei freelancer e dei lavoratori con contratti precari rispetto alle pressioni degli editori e dei proprietari dei media, compromettendo l'autonomia dei giornalisti (Siapera 2015). Al contempo, la mancanza di occupazioni stabili e durature per i giornalisti sta sfociando verso tendenze imprenditoriali nel giornalismo (Jarvis 2010), per cui i giornalisti sono incoraggiati a ipotizzare nuove start-up giornalistiche, ma anche a ritenere loro stessi un marchio da gestire e vendere. Bruns (2012) riporta che i giornalisti australiani possono agire su Twitter come "marchi personali", ritwittando gli articoli scritti per le organizzazioni informative tramite i loro account personali. In termini più strutturali, Siapera e Spyridou (2012) ritengono che i network costruiti dai giornalisti sui social media siano una forma di capitale sociale, che può essere trasformato, in certe circostanze, in altre forme di capitale. Nel costruire e mantenere viva un'ampia rete di amici e di follower su Facebook e su Twitter, i giornalisti possono migliorare la propria posizione sul campo. La traiettoria di Andrew Sullivan ne è un buon esempio. Sullivan era uno dei primi blogger politici degli Stati Uniti all'inizio degli anni 2000, scrivendo per il The Dish. Sulla base della sua notorietà e del suo successo legati al blog, gli venne offerta la posizione di redattore dell'Atlantic Monthly, dove si dice che abbia contribuito a un aumento dell'attività del circa 30\% (Perez-Pena 2008). Mentre non tutti i giornalisti presenti sui social media godono di una tale notorietà ed alta visibilità, costruire e mantenere una rete di follower costituisce una parte sempre più importante del loro lavoro, e spesso offre una fonte di stabilità in un ambiente altrimenti movimentato caratterizzato da precarietà ed impieghi a breve termine. 
Le iniziative imprenditoriali, nonostante il picco di tendenza, non si sono rivelate particolari opportunità per il giornalismo. Nonostante l'imprenditoria sembri offrire una possibile via per i giornalismo, non tutto è ciò che sembra. Per intellettuali come Jeff Jarvis (2009), il futuro del giornalismo è imprenditoriale, non istituzionale, come da lui stesso affermato: "Credo che i giornalisti debbano diventare imprenditori. ... Devono sentire e servire il mercato. Devono lavorare con innovatori. Devono intravedere un futuro diverso per il giornalismo, anche migliore del suo passato". Come precedentemente affermato, le strutture gerarchiche ed i ritmi rigidi danno la priorità ad una concezione del mondo ed un'autonomia giornalistica limitata. Inoltre, la precarietà e l'insicurezza lavorativa significano che i giornalisti devono essere più consapevoli delle opportunità di mercato per loro stessi. L'imprenditoria sembra quindi essere in linea con lo spirito del tempo: "Non odiare i media, sii tu stesso i media", pare dire.

In ogni modo, il manifesto per il giornalismo imprenditoriale di Jarvi pone l'accento sul profitto, non sulla qualità giornalistica o sul giornalismo inteso come servizio pubblico. Inoltre, le critiche che Jarvis ed altri muovono in questo campo nei confronti della condizione del giornalismo di oggi sembrano colpire ingiustamente $\mathrm{i}$ giornalisti stessi più che identificare i parametri più ampi che determinano il lavoro di questi ultimi. Ma forse uno degli aspetti più problematici del "journopreneurialsm" (Prein 2014) è che i giornalisti dovranno occuparsi di tutti gli aspetti del business, trovare capitali a rischio e rispondere agli investitori, tutto ciò mentre praticano il "giornalismo innovativo". La pressione che grava su un'occupazione già ora molto stressante è immensa. Per altri commentatori, i problemi sono addirittura più profondi. Dean Starkman (2011) parla del consenso del "futuro delle notizie" ("future of news", FON), capeggiato da Jarvis, Shirky ed altri, il quale sostiene l'imprenditoria a spese del giornalismo. Il valore chiave del giornalismo consiste nel servire l'interesse pubblico, ma, nella propria ricerca del profitto e nella spinta verso un maggiore impegno nei confronti dei lettori, l'imprenditoria perde tali tratti. In un certo senso, il giornalismo imprenditoriale sembra essere una specie di stenografia per il capitalismo neoliberale e l'innovazione è rivolta, più che verso un servizio nei confronti della società, verso il profitto privato. Il giornalismo diventa soltanto un ulteriore servizio, un altro bene da acquistare e da vendere tra individui, e non funge più da mediatore tra il potere e il cittadino. Da questo punto di vista, l'imprenditoria non offre una soluzione per la sostenibilità del giornalismo. Come evidenziato da Pein (2014, non numerato), l'imprenditoria orientata verso la tecnologia non riguarda il giornalismo o i giornalisti: "Le sue priorità consistono nell'automatizzazione, nella 
standardizzazione e nella deprofessionalizzazione; far fare tutto ai robot, e ciò che i robot non riescono fa fare, lasciarlo ai c.d. 'Redditors'".

Uno sguardo pragmatico all'evoluzione delle varie iniziative imprenditoriali ci mostra un quadro variopinto. Vi sono alcuni successi veri, soprattutto il danese De Correspondent, ma la maggior parte delle iniziative giornalistiche imprenditoriali che ha avuto successo deve il proprio risultato positivo al sostegno di potenti imprese. Ezra Klein, l'ex caporedattore del The Washington Post, per esempio, ha lanciato Vox, una start-up giornalistica che pone l'accento sul "giornalismo innovativo". Vox ha ottenuto un buon successo, con un gruppo di lettori fedeli e follower sui social network, ma è spalleggiato da Vox Media che detiene anche SB Nation (sport), the Verge (tecnologia), Polygon (giochi), Curbed (compravendite immobiliari), Eater (mangiare e bere) e Racked (shopping/lifestyle). Vox Media ha ottenuto 70 milioni di dollari grazie alle speculazioni ed annovera tra i propri investitori Comcast e Accel Partners. In altre parole, stiamo parlando di un investimento considerevole da parte di di finanziatori che si aspetteranno un rientro considerevole. Uno sviluppo interessante consiste nel fatto che Vox Media è uno dei partner di lancio di Apple News, una "native reader app" (Warren 2015), nata grazie ad un'altra sinergia tra le imprese dei media e di internet. Sembra che i milionari di internet si stiano rapidamente muovendo verso il settore dell'informazione, con Pierre Omidyear di Ebay e Jeff Bezos di Amazon che hanno, rispettivamente, investito nel The Intercept di Glenn Greenwald e nel The Washington Post. Storyful, fondato come start-up dall'ex giornalista di RTE (Irlanda) Mark Little, ha ottenuto un successo considerevole e si è costruito una significativa reputazione come agenzia di notizie digitale. Ma, all'incirca nel 2012, è entrato in difficoltà economiche, operando in perdita finché venne rilevato da News Corp nel dicembre del 2013 (Byrne 2014). Il coinvolgimento di grandi imprese nell'ambito del giornalismo digitale mostra che le idee romantiche di imprenditori da quattro soldi con grandi idee in grado di fare soldi sono molto distanti dalla realtà. Gli investimenti di successo hanno fatto affidamento al saper fare ed alla reputazione di giornalisti affermati sostenuti da finanziamenti provenienti da grandi imprese. Sembra che nel campo di internet l'informazione sia di nuovo divenuta un affare commerciale.

Nel concludere il capitolo dedicato alla produzione del giornalismo, appare chiaro che molto è cambiato e che la produzione del giornalismo affronta una serie di sfide importanti. Le principali di esse sono: trovare un nuovo modello di business e di guadagno che assicuri la sostenibilità del giornalismo senza comprometterne i valori e l'etica; in secundis, raggiungere un nuovo accordo tra giornalisti ed editori che determini chiaramente i diritti ed i doveri di entrambe le controparti nei confronti dell'altra, tute- 
lando l'integrità del giornalismo indipendentemente dalla condizione di impiego.

\subsection{Forma e contenuto}

Questo capitolo si occupa delle sfide che internet rappresenta per la forma e per il contenuto del giornalismo. Per iniziare, il termine "forma" è qui utilizzato per fare riferimento ai modi in cui è organizzato il contenuto, mentre "contenuto" richiama i soggetti e la sostanza del giornalismo e le loro proprietà qualitative. Il contenuto precede la forma, perché ne è parte costitutiva. Secondo Adorno (2003 [1970]), la forma è il contenuto sedimentato estratto dalla realtà. Il significato viene trasmesso dalla forma stes$\mathrm{sa}$, in altre parole tramite una specifica disposizione del contenuto. Si tratta di una distinzione necessaria perché dimostra che la forma ed il contenuto coesistono in un rapporto di tensione: la forma è una costante in quanto rimane la medesima in trasposizioni concrete differenti. Per esempio, gli articoli di cronaca, gli editoriali e gli articoli di fondo esistono come forme in diversi giornali. Dall'altro lato, il contenuto non è sempre lo stesso. Per esempio, l'editoriale di un giornale è diverso da quello di un altro anche se entrambi trattano lo stesso argomento. In altre parole, il significato deriva dalla stabilità della forma e dalla novità del contenuto: un modo (formale) di organizzare il contenuto consente ai lettori di comprendere ciò che stanno leggendo. La tensione si basa sulla tendenza, per il contenuto cristallizzato, di ottenere nuove forme. Ciò è produttivo/creativo e necessario per il giornalismo o per qualsiasi altro modo di produzione dei contenuti per rimanere rilevante. Una delle sfide per il giornalismo nell'era dei social media consiste nel riconciliare le nuove forme emerse con i contenuti che formano il giornalismo e le notizie. Inoltre, come vedremo, il crescente ruolo centrale ricoperto dai social media nel divulgare i contenuti, e di conseguenza nell'imporre una singola forma su di essi, comporta implicazioni importanti per il significato di tali contenuti. La trattazione è qui organizzata come segue: la prima parte si occupa del contenuto, della sostanza del giornalismo, ponendo l'accento su due tipi di fattori che potrebbero coinvolgerlo: fattori organizzativi e legati ai media. Essi vengono utilizzati in modo euristico al fine di guidare la trattazione e non corrispondono a categorie distinte chiaramente delimitate. Infatti, i fattori mediatici non possono operare al di fuori dei contesti organizzativi. Nondimeno, la distinzione qui effettuata è utile per evidenziare i vari impatti dei diversi fattori. La seconda parte si occupa delle nuove forme del giornalismo, trattando più 
nello specifico il giornalismo dei dati. La parte finale identificherà alcune delle tensioni e delle sfide emergenti.

\subsubsection{Fattori organizzativi}

I fattori organizzativi che riguardano i contenuti si riferiscono soprattutto all'impatto, potenziale e reale, che i cambiamenti nella produzione del giornalismo potrebbero provocare sulla sostanza dello stesso. Essi seguono dall'analisi precedentemente esposta ed includono l'intensificazione del circuito informativo e la costante richiesta di guadagni e di modelli di guadagno.

Come visto precedentemente, il circuito dell'informazione attivo 24 ore su 24 e 7 giorni su 7 impone ai giornalisti pressioni considerevoli affinché producano notizie di continuo. In ogni modo, tale continua corsa alla pubblicazione non comporta necessariamente una maggiore ricerca di notizie. Inoltre, $\mathrm{i}$ tempi stretti significano che i giornalisti non sono più in grado di verificare ciò che stanno pubblicando. L'intenso circolo dell'informazione significa, insieme ad un alto numero di licenziamenti ed un crescente affidamento al lavoro free-lance, che la verifica dei fatti ed il controllo sulla qualità dell'informazione hanno sofferto in questo nuovo sistema. Ma forse uno degli sviluppi più pericolosi in termini di contenuti è il c.d. "churnalism". Secondo Harcup (2015: 8) il termine venne utilizzato per la prima volta dal giornalista della BBC Wassem Zakir, che lo usò indicando la mancanza di servizi giornalistici originali e la prevalenza degli articoli a sfondo pubblicitario. Come affermato da Zakir, i giornalisti "ricevono la copia che arriva via cavo ed i reporter ne producono in serie, modificandoli e magari aggiungendo qualche citazione locale" (in Harcup, 2015: 8). Mentre Zakir si riferiva soprattutto all'influenza dei comunicati stampa, il c.d. "churnalism" si è allargato nell'era dei social media, portando ad un giornalismo uniforme del "copia e incolla", in cui si ripetono sempre i medesimi articoli.

La ricerca sembra confermare ciò. Redden e Witschge (2009) hanno scoperto che le varie aziende del settore dei media hanno riportato le stesse tematiche in modo molto simile. Hanno rilevato alti tassi di omogeneità, le stesse immagini, le stesse citazioni e le stesse descrizioni in molti giornali e siti internet del Regno Unito. Hanno anche trovato alti livelli di riciclo interno con piccole variazioni nei titoli e nel testo. Redden e Witschge attribuiscono ciò alla pressione che grava sui giornalisti affinché producano contenuti per varie piattaforma ed al crescente affidamento ai circuiti dell'informazione ed alle agenzie di marketing. In alcuni casi tale ripetizione 
dei contenuti può essere attribuita ad una contraddizione tra la natura della notizia, che spesso consiste di un evento unico con sviluppi che seguono lentamente e nel coso di giorni e mesi, e la natura del circolo dell'informazione attivo 24 ore su 24 e 7 giorni su 7 e la continua richiesta di nuovi contenuti. Inoltre, i licenziamenti e l'affidamento ai free-lancer pagati a cottimo significa che ci sono meno giornalisti che seguono una notizia e che più notizie sono più care.

Ciò implica che il contenuto che vediamo on-line non sempre è originale. In ogni modo, non si tratta di un fenomeno necessariamente nuovo. Il problema è che il connubio tra il giornalismo e internet avrebbe dovuto democratizzare il giornalismo (Gillmor 2004), innovarlo e sbaragliare i valori dell'informazione consolidati e diversificare i tipi di notizie da pubblicare. Al contrario, abbiamo il c.d. "churnalism" e le notizie mancano talmente tanto di originalità che potrebbe scriverle una macchina. In realtà esistono già i robot che scrivono articoli. Il seguente articolo, pubblicato sul Los Angeles Times, è stato scritto da "Quakebot", un algoritmo scritto da Ken Schwencke, un giornalista e programmatore:

A shallow magnitude 3.0 earthquake was reported Thursday afternoon one mile from

Brea, according to the U.S. Geological Survey. The temblor occurred at 3:35 p.m. Pacific

time at a depth of 0.6 miles.

According to the USGS, the epicenter was two miles from La Habra, two miles from

Rowland Heights, and four miles from Placentia.

In the last 10 days, there have been no earthquakes of 3.0 or greater centered nearby.

This information comes from the USGS Earthquake Notification Service and this post was created by an algorithm written by the author (Quakebot 2014).

Un sisma superficiale di magnitude 3.0 è stato segnalato giovedì pomeriggio alla distanza di un miglia da Brea, secondo il Servizio geologico degli Stati Uniti (USGS). Il terremoto si è verificato alle ore 15:35 ora del Pacifico ad una profondità di 0,6 miglia.

Secondo l'UGSG, l'epicentro si trovava a due miglia di distanza da La Habra, due miglia da Rowland Heights e quattro miglia da Placentia.

Negli ultimi dieci giorni non si erano verificati sismi di magnitude 3.0 o superiore. Queste informazioni provengono dall'USGS Earthquake Notification Service e la presente notizia è stata creata da un algoritmo scritto dall'autore (Quakebot 2014). 
Schwencke afferma che l'intero processo è durato tre minuti (Oremus 2014). L'idea di fondo consiste nel fatto che gli algoritmi possano svolgere il lavoro di "churning", mentre i giornalisti possono liberare un po' di tempo per affrontare le questioni più complesse. In ogni modo, è probabile che gli algoritmi verranno utilizzati per scrivere sempre più articoli, per esempio comunicati stampa che si adattano in automatico, ma ciò potrebbe non significare ex se che i giornalisti scrivano più articoli. Nonostante i robot non siano in grado di sostituire i giornalisti e di scrivere articoli originali, essi possono produrre contenuti in serie, in tal modo inondando il web di contenuti non originali che vengono occasionalmente abbelliti con grafici o figure e che giungono ai lettori. Più aumentano le riproduzioni in serie, meno sono in grado i lettori di distinguere tra i contenuti, distinguere ciò che è vero da ciò che non lo è, distinguere gli originali dalle copie, ecc. Ciò impone a sua volta sfide serie alla credibilità del giornalismo e, paradossalmente, accresce ancor più l'importanza del giornalismo inteso come attività originale ed accurata.

Mentre il c.d. "churnalism" è da attribuirsi, in parte, alla pressione temporale imposta dal circolo dell'informazione attivo 24 ore al giorno 7 giorni su 7, la richiesta di guadagni si è rivelata più pericolosa per i contenuti giornalistici, dando adito ad un modo di scrivere finalizzato al miglioramento della performance sui motori di ricerca, al c.d. clickbaiting ed alla pubblicità nativa. La sovrabbondanza di contenuti negli ambienti on-line ha alimentato l'ascesa dei motori di ricerca e di algoritmi basati sugli stessi che forniscono risultati su una base data e li ordinano secondo un certo ordine di scala o di importanza. Nello specifico, l'algoritmo di Google, PageRank, si basa un algoritmo che calcola l'importanza di un sito web sulla base del numero di link ottenuti da altri siti, mentre anche il livello di questi siti viene preso in considerazione. Quando quindi gli utenti cercano una parola, Google presenta i risultati sulla base dell'importanza o del livello dei siti internet ove tale parola appare (nonostante vengano anche presi in considerazione una serie di altri fattori, quali la posizione, la cronologia delle ricerche, ecc.). PageRank è diventato un algoritmo enormemente complesso la cui struttura esatta è segreta e tutelata da brevetti. Dal punto di vista dei giornalisti e dei nuovi siti, l'esigenza di indirizzare i lettori verso i loro siti al fine di attirare gli inserzionisti pone loro la pressione di scrivere in modo tale da catturare gli algoritmi di ricerca.

L'ottimizzazione dei motori di ricerca include quattro elementi cardine: la ricerca per parola chiave, l'indicizzazione dei motori di ricerca, l'ottimizzazione on-page e l'ottimizzazione off-page (Malaga 2008). In uno studio etnografico condotto nel Regno Unito, Dick (2011) ha scoperto che l'ottimizzazione dei motori di ricerca sta diventando un aspetto sempre più im- 
portante dell'informazione on-line, con impatto sul contenuto delle storie scelte per la pubblicazione e sul modo in cui esse sono strutturate. Per esempio, una tecnica del genere consiste nell'inserire parole chiave di tendenza nei titoli o in generale nel riscrivere le storie utilizzando parole search-friendly, trattandosi di parole che gli utenti potrebbero utilizzare durante la ricerca di un articolo. Dick (2011: 475) giunge alla conclusione che l'ottimizzazione dei motori di ricerca sta cambiando gli standard giornalistici "nell'interesse di un terzo commerciale, giudice nella distribuzione on-line: Google. Sta emergendo un nuovo conformismo nel linguaggio e la c.d. 'aboutness' di notizie on-line, mediata da Google. Google si posiziona tra le parole utilizzate dal pubblico dei lettori e dai giornalisti allo stesso modo, regolando implicitamente il 'mercato delle idee' per come funziona". Si tratta di una critica significativa che deve essere presa in considerazione.

La corsa ai guadagni nel digitale determinata, in realtà, da Google e dagli altri principali social media, ovvero Facebook e Twitter, ha anche portato all'ascesa del c.d. giornalismo "clickbait". Tale pratica si riferisce alla redazione di titoli che inducono i lettori a cliccarvi sopra ed a condividere l'articolo. Uno degli esempi più famosi del c.d. giornalismo "clickbait" fu l'articolo di Samantha Brick nel Daily Mail dal titolo "There are downsides to looking this pretty: Why women hate me for being beautiful" "Essere belle ha dei lati negativi. Perché le donne mi odiano per la mia bellezza"). L'articolo di Brick è un esempio riuscito di clickbait perché è allo stesso tempo insulso e controverso - adatto a provocare sdegno nei confronti dell'autore, ma non sufficientemente controverso per criticare il Daily Mail per averlo pubblicato. Nel momento di punta, l'articolo riceveva quattro commenti al minuto ed in pochi giorni ha raccolto sei milioni di visualizzazioni. Le stime sui guadagni variano tra le 37.500 e le 100.000 sterline (Smith 2012). Soprattutto ha aiutato il Daily Mail a consolidarsi come possibile fonte di contenuti virali, di conseguenza aumentandone potenzialmente il valore aggiunto.

Il "clickbait" viene altresì frequentemente utilizzato dai siti di informazione e di commenti internet quali Upworthy. Un tipico modo per distinguere il clickbait consiste nell'uso della citazione precedente come esca (Blom e Hansen 2015). Questo esempio di Upworthy lo mostra chiaramente: "Calling them 'girls' was their first mistake. What happened next is a great moment in fierceness" ("Chiamarle 'ragazze' è stato il loro primo errore. Quello che è accaduto poi è stato un grande attimo di ferocia"), oppure "His first 4 sentences are interesting. The 5th blew my mind" ("Le sue prime quattro frasi sono interessanti. La quinta mi ha completamente scossa"). I titoli mirano a catturare l'attenzione dei lettori e li convincono a cliccare 
su un link per saperne di più. Cliccando, essi vengono registrati come lettori e, di conseguenza, aumentano il valore del sito internet. In ogni modo, vi è una crescente reazione contro il clickbait, ed i lettori si sentono frustrati e manipolati da titoli del genere mentre in altri casi essi ritengono che l'informazione richieda un trattamento più serio e meno scandalistico. Per esempio, nel gennaio del 2014, il telegiornale della BBC ha twittato la seguente frase: "14-year-old girl stabbed her little sister 40 times, police say. The reason why will shock you" ("Ragazzina quattordicenne pugnala la sorellina minore con 40 coltellate. Il motivo vi lascierà sconvolti”). La reazione di Twitter a ciò faceva riferimento all'identità della CNN quale fonte mediatica legittima, che aveva danneggiato sé stessa e la propria credibilità, e, in secondo luogo, intorno all'etica del giornalismo serio che dovrebbe trattare le persone con rispetto e non scandalizzare. Recentemente Facebook ha deciso di adottare misure contro il clickbait in quanto non voleva che il clickbait "sovrastasse i contenuti degli amici e delle pagine che realmente interessano alle persone" (Chowdhry 2014) modificando il suo algoritmo. A questo punto è importante notare che il clickbait rappresenta una tipologia specifica di adattamento dell'informazione e dell'industria mediatica all'ambiente dei social media. Mentre i media d'informazione vantano una storia di tentativi di attirare i lettori tramite titoli in grado di catturarne l'attenzione, le tecniche di clickbait e di organizzazione dei motori di ricerca mostrano un adattamento specifico dei contenuti alle richieste dei social media. E, come evidenziato da Dick (2011), ciò non avviene nell'interesse del pubblico o del giornalismo stesso, bensì per resistere al nuovo ecosistema sempre più controllato dai social media e dai loro algoritmi. La sfida qui rappresentata per il giornalismo consiste nel riconciliare la cultura dei $s o-$ cial media con le proprie etiche ed i propri valori.

In ogni modo, l'onnipresente richiesta di sopravvivenza all'interno di un tale ambiente spesso conduce a pratiche e decisioni discutibili. Una di queste è il c.d. "native advertising". Si tratta della prassi secondo cui un'inserzione commerciale viene inserita in un sito di informazione utilizzandone il medesimo formato in modo da mascherare il fatto che si tratta di un contenuto pubblicitario a pagamento. Di nuovo, questa pratica ha una sua storia al di fuori dall'ambiente digitale, ma è on-line che ha acquisito nuova importanza. Una delle ragioni principali di ciò è la ben risaputa tendenza dei lettori di evitare le pubblicità. Le inserzioni digitali registrano, infatti, numeri di click notoriamente bassi (Volpe 2013), mentre la popolarità dei c.d. "ad blocker" sta ponendo sfide senza precedenti agli inserzionisti. La c.d. pubblicità nativa rappresenta quindi un mezzo con il quale ovviare a tali problematiche, e tale è la ragione per la quale sta divenendo sempre più popolari e per la quale la si trova in molti siti internet dell'informazio- 
ne. Ne fanno uso Buzzfeed, the Atlantic, MailOnline, The New York Times e molti altri. Questa forma di inserzioni pubblicitarie ha suscitato controversie perché trae in inganno i lettori, i quali credono, in buona fede, che si tratti di articoli giornalistici. EMarketer (2014) riporta una ricerca di mercato che dimostra che, negli Stati Uniti, circa il 31\% dei lettori non è in grado di distinguere tra il giornalismo e la pubblicità nativa, mentre nel Regno Unito il 49\% dei lettori ritengono gli articoli pubblicitari ingannevoli. Alla lunga, lo scetticismo dei lettori potrebbe espandersi a tutti i contenuti, compromettendo, di conseguenza, seriamente la credibilità del giornalismo. La pubblicità nativa rappresenta un'altra forma di adattamento del contenuto all'ecosistema dei media attuale, ma si tratta di una forma con effetti potenzialmente molto dannosi.

\subsubsection{Fattori legati ai media}

Mentre il fenomeno del c.d. "churnalism" potrebbe essere il risultato di adattamenti organizzativi all'aumento di competizione per accaparrarsi i lettori e della continua ricerca di un numero maggiore di contenuti, in questo capitolo l'accento è posto sulle peculiarità dei nuovi media che coinvolgono i contenuti in modi specifici. Queste caratteristiche possono essere intese come "affordances" ("inviti all'uso"), si tratta di una caratteristica specifica di determinati media che consentono o limitano l'uso a determinati utenti. Il concetto di tali "affordances" deriva dalla psicologia percettiva ed è stato utilizzato come mezzo con il quale comprendere come le persone percepiscono, comprendono ed utilizzano l'ambiente che li circonda (Gibson 2014 [1979]). Hutchby (2001) ha definito tale concetto un'utile correzione della tendenza verso il determinismo tecnologico, sostenendo che le "affordances" puntano al modellamento reciproco delle tecnologie e delle persone. Nello specifico, Hutchby definisce le "affordances" tecnologiche gli "aspetti funzionali e relazionali che fungono da cornice, pur non determinandole, alle possibilità per un'azione 'agentica' ('agentic') in relazione ad un oggetto" (2001: 444). Le "affordances" si trovano da qualche parte tra gli oggetti ed i percettori/utenti: si tratta di proprietà funzionali delle tecnologie, ma meno che non vengano recepite e utilizzate dagli utenti, diventano latenti ed infine si atrofizzano. Per fare qualche esempio di "affordances", possiamo dire che i telefoni cellulari consentono la mobilità e la comunicazione, internet consente la connettività globale, Twitter permette una forma di comunicazione breve ed immediata con molte altre controparti. Potrebbero esservi anche "affordances" annidate: per esempio, l'applicazione mobile di Twitter ed il suo affidamento a inter- 
net significa che offre anche le "affordances" di quest'ultimo. Data la posizione mediata tra la tecnologia e gli utenti, è probabile che le diverse categorie di utenti percepiscano "affordances" differenti: un giornalista percepisce "affordances" diverse rispetto ad un politico, un insegnante, un esperto di marketing o un adolescente.

Lister et al. (2009) fanno riferimento ad una serie di caratteristiche dei nuovi media che possono poi diventare un punto di partenza per pensare alle "affordances". Esse includono (i) la digitalità, in cui tutto il contenuto mediatico è ora codificato allo stesso modo e può quindi essere immediatamente trasferito da una piattaforma mediatica all'altra - ciò ha alimentato direttamente la convergenza di tutti i media; (ii) la multimedialità, in cui i giornalisti ora scrivono articoli che includono audio, video e testi; (iii) l'interattività, $o$ la possibilità di una comunicazione bidirezionale più o meno immediata, che ha portato alla nascita dei commenti e al coinvolgimento dei lettori nel contenuto dell'informazione; (iv) l'ipertestualità, che si riferisce alla capacità di introdurre collegamenti al testo addentrando i lettori maggiormente nella tematica che stanno leggendo - ciò introduce un certo dinamismo nel contenuto, che può ora essere esteso fino all'infinito; (v) e infine la connettività, gli utenti possono collegarsi tra di loro e divenire parte della stessa rete, formando una comunità di lettori. Tutti questi fattori hanno avuto un impatto significante sul contenuto giornalistico che deve ora operare in condizioni molto diverse. La multimedialità, l'interattività e l'ipertestualità hanno un ruolo centrale nella letteratura, evidenziando i nuovi modi di scrivere e di narrare i contenuti agli utenti, rendendo il tutto più dinamico ed abbinando al testo informazioni visive e uditive, offrendo contemporaneamente ai lettori l'opportunità di partecipare. Forse uno dei pezzi del giornalismo più significanti emersi negli anni recenti come paradigma di eccellenza che dimostra ciò che si può raggiungere nel campo dei nuovi media, è "Snow fall: the avalanche of Tunnel Creek", articolo pubblicato nel 2012 sul sito internet del The New York Times. Il pezzo venne insignito del Premio Pulitzer per il miglior articolo nel 2013, avendo consentito ai lettori di "percepire la neve e toccare il panico" che aveva colto gli sciatori intrappolati sotto la valanga. Il pezzo integra senza interruzioni testo, video, immagini e grafiche per narrare non solo la vicenda della valanga specifica, ma anche la fisionomia delle valanghe. È stato acclamato dalla critica e ha ottenuto commenti favorevoli e apprezzamenti da parte dei lettori, alcuni lo hanno accolto come il futuro del giornalismo (Greenfield 2012). Apparentemente, "to snowfall" è ora diventato un verbo in lingua inglese utilizzato in molte redazioni come abbreviazione per una narrazione multimediale efficace. 
L'articolo sullo "snow fall" fu un caso eccezionale, in cui gli sforzi tecnologici si fondono in maniera organica con gli elementi giornalistici della narrazione, ma, nella maggior parte dei casi, sussiste l'esigenza di adattazione considerevole e di attenzione verso le "affordances" di piattaforme mediatiche specifiche. Dando, per esempio, uno sguardo alle "affordances" di Twitter, la brevità, l'immediatezza, la connettività e gli hashtag, possiamo vedere il tipo di giornalismo che emerge. I tweet possono essere costanti ma devono racchiudere il messaggio in 140 caratteri. Costringe i giornalisti a scrivere quasi solo titoli. Ma in aggiunta a ciò, le "affordances" di Twitter e le pratiche degli utenti hanno condotto ad uno specifico tipo di pratica giornalistica di Twitter: Lasorsa et al. (2012) hanno rilevato che i giornalisti che lavorano per i media mainstream degli Stati Uniti tendono a twittare più opinioni ed a fornire certi standard di responsabilità e di trasparenza sulle loro pratiche collegando e condividendo/"ritwittando" altri contenuti. Hermida (2013) ricorda che, all'inizio, alcuni media impostarono un invio automatico dei loro titoli su Twitter, prassi che però non funzionò perché gli utenti si mostrarono poco interessati a questi tipi di tweet. Evidentemente, affinché il giornalismo funzioni su Twitter, deve conformarsi alla cultura del mezzo che è emersa da tale rete di affordances tecnologiche e dalla prassi seguita dalle utenti. Alcune di queste pratiche emergenti e che hanno riguardato i contenuti in modo più o meno diretto, inclusa l'ascesa delle notizie emotive (Papacharissi e Oliveira 2012), le quali si riferiscono alla combinazione di notizie con punti di vista e opinioni. Vi è anche una personalizzazione, ovvero l'assunzione di una prospettiva personale, dibattito ed impegno diretto, facendo riferimento all'idea che non basta scrivere e attendere che gli altri si limitino a leggere. Ai giornalisti viene ora richiesto di rispondere, di condividere/'ritwittare', commentare e replicare ai lettori e ad altri. Devono, inoltre, mettersi in rete con altri soggetti, seguirli ed infine utilizzare hashtag che riportano e che creano collegamenti con la notizia principale. Un ultimo aspetto importante che riguarda i contenuti su Twitter e su altri social media fanno riferimento al risultato della pressione di pubblicare le notizie immediatamente. Un modo per fare fronte a ciò consiste nel condividere l'incertezza e nel rendere ripercorribile il processo di creazione della notizia. Si tratta della strategia seguita da Andy Carvin di NPR (National Public Radio, Stati Uniti), il quale è stato il primo ad adottare una strategia del genere postando tweet sui nuovi sviluppi e chiedendo agli utenti di verificarli (Hermida et al. 2014). Come sostenuto da Hermida et al. (2014), il giornalismo su Twitter sorge come prassi di autenticazione e interpretazione di "flussi di consapevolezza sociale".

Per concludere il capitolo sul contenuto, le sfide principali qui presenti per il giornalismo comprendono innanzitutto l'adattamento reciproco del- 
le routine organizzative alle prassi ed alle esigenze dei social media e, in secundis, l'adattamento reciproco del contenuto giornalistico e delle "affordances" tecnologiche. Questi fattori possono creare tensioni ma, come dimostrato dall'articolo dello "snow fall" e dai casi di Andy Carvin, esiste un potenziale positivo molto grande.

\subsection{La forma}

Come precedentemente menzionato, da una tensione produttiva tra i nuovi contenuti e le vecchie forme sono sorte nuove forme di giornalismo. Da questo punto di vista, esse sono sorte anche perché i nuovi media hanno consentito la produzione e la circolazione di nuovi tipi di contenuti in nuovi modi cosicché le vecchie forme del giornalismo dovettero, di conseguenza, adattarsi. Nel comprendere i cambiamenti coinvolti e le tensioni e le sfide che essi pongono, l'approccio qui seguito si basa sull'esame di una serie di casi. Identificare e schematizzare le nuove forme è molto difficile, soprattutto in quanto molte delle nuove forme sono ibride, ovvero che si "prendono in prestito" e si "rubano" a vicenda. L'euristica qui utilizzata per identificare e analizzare è la seguente: come producono e organizzano le nuove forme i vari elementi costitutivi e come si relazionano con il pubblico. La trattazione si snoderà sull'esame di due casistiche, il c.d. live blogging e il c.d. giornalismo dei dati quali due influenti nuove forme di giornalismo.

\subsubsection{Il c.d. "live blogging"}

Il c.d. "live blogging" sta sempre più diventando la forma prediletta per la narrazione di eventi in diretta, eventi sportivi e per le notizie in tempo reale. È stato definito "un singolo post su un blog su un argomento specifico al quale viene progressivamente aggiunto un contenuto per tempo in un determinato periodo che varia da mezz'ora a 24 ore" (Thurman e Walters 2013: 83). Si iniziò circa nel 1999, quando fu il The Guardian a introdurre tale prassi, ma venne usata soprattutto per gli eventi sportivi. Il c.d. "live blogging" prevede altresì l'incorporazione di elementi multimediali e di collegamenti ad altre pagine. È una forma giornalistica che si presta per diversi tipi di contenuti e ha avuto successo tra gli utenti, i quali apprezzano la narrativa continua, che si sviluppo, il ché tiene conto anche delle abitudini dei lettori nel senso in cui essi leggono le notizie su internet. 
Un paradosso che riguarda il c.d. "live blogging" è dato dal fatto che la maggior parte di esso non avviene sul campo. Come affermato da alcuni giornalisti in Thurman e Walters (2013), è più semplice scrivere il blog in ufficio attingendo a fonti televisive o altri media grazie alla connessione wi$f i$ che sul campo utilizzando soltanto il proprio pc e facendo affidamento ad una connessione internet instabile. D'altro canto, siccome l'utilizzo mobile si sta diffondendo, molti tweet vengono scritti sul campo documentando un avvenimento in diretta. Il c.d. "live blogging" che avviene in ufficio è da considerarsi meglio una mediazione o una cura delle informazione più che una narrazione originale, mentre il 'twittaggio' sul campo può essere visto come proto-riporto, narrando gli avvenimenti in diretta.

Gli elementi narrativi del c.d. "live tweeting" includono una vicenda continuata che si evolve col passare del tempo e che viene descritta da molteplici punti di vista e prospettive. Il c.d. "live blogging" vuole essere colloquiale e spesso contiene notizie non verificate con avvertimenti. In considerazione di ciò si tratta di una forma giornalistica più libera e più trasparente. Il c.d. "live blogging" trasmette il tempo reale e la confusione delle notizie e vicende in evoluzione e la tempestività delle notizie, mostrando quando è caotica la realtà e spesso illustrando le decisioni che i giornalisti prendono in termini di cosa viene ritenuto degno di cronaca e cosa no. In questo modo, il c.d. "live blogging" rimuove i giornalisti dal piedistallo della fonte esperta, onnivedente e onnisapiente fonte di informazione. Rappresenta altresì un adattamento al frenetico, ipercollegato ambiente dei social network. Beckett (2010) ha trattato il fenomeno in parola definendolo la nuova prima pagina.

Dall'altro lato, tale forma giornalistica è stata accusata di essere responsabile della "morte del giornalismo" (Symes 2011) perché contiene una combinazione di informazioni rilevanti ed irrilevanti senza differenziazioni di alcun tipo eccetto l'elemento temporale: la prima notizia è la più recente. Il tipo di competenza che i giornalisti possono introdurre nella vicenda separando le informazioni utili da quelle meno importanti e riordinando la confusione di notizie si perde nel c.d. "live blogging". Anderson (2011) fa riferimento a ciò affermando che tale prassi sta causando un sovraccarico di informazioni tramite "fiumi di notizie" (Anderson 2011). Alla fine, i lettori potrebbero confondersi ancor più ed essere meno informati a causa del c.d. "live blogging". Inoltre, scrivendo le informazioni sul blog quando si creano, tale prassi potrebbe indebolire gli standard di verifica (Petrie 2011), malgrado gli avvertimenti e le liberatorie dalla responsabilità pubblicate dai giornalisti. La questione centrale che riguarda il c.d. "live blogging" (e il c.d. "live tweeting) rimane la seguente: si tratta di giornalismo? Anderson (2011) suggerisce che potrebbe forse essere considerato 
giornalismo se non consistesse solo nella raccolta di informazioni rilevanti, bensì ne provvedesse anche il contesto e la cura, in altre parole se prevedesse un processo di informazione più articolato invece di elencare singoli fatti.

Accettando le critiche formulate contro questa forma giornalistica, possiamo vedere che essa si adatta alla frenesia ed all'ambiente dinamico dei social media. Al contempo, affronta ancora la sfida di adattare con successo le regole ed i valori del giornalismo alla prassi del c.d. "live blogging" o "live tweeting".

\subsubsection{Il giornalismo dei dati}

Il giornalismo dei dati si riferisce all'uso dei dati per la redazione degli articoli e per la comprensione degli sviluppi. Si tratta di una forma ibrida che include l'analisi statistica, la scienza informatica, la visualizzazione ed il web design ed il riporto di informazioni (Coddington 2015). Il giornalismo dei dati è considerato uno sviluppo chiave del giornalismo, in quanto è visto come valore aggiunto allo stesso (Lorenz 2012). Il giornalismo dei dati non si riferisce alla descrizione di avvenimenti in diretta, bensì mira a dare senso a un contesto più ampio utilizzando le informazioni grezze ed analizzando i numeri. A differenza del c.d. "live blogging", che è a portata di quasi tutti, il giornalismo dei dati richiede conoscenze nel campo della statistica abbinate a tecniche di visualizzazione dei dati. In tal senso, questa nuova forma di giornalismo reintroduce l'elemento della competenza, ma non si tratta più di una competenza redazionale o della ricerca di informazioni, bensì nella lettura di numeri e di competenze di natura matematicoscientifica. Il giornalismo dei dati è una delle nuove forme più promettenti perché combina la logica del giornalismo (l'importanza, la rilevanza della notizia, l'analisi) e le logiche dei nuovi media (l'informazione, i big data così come l'open source).

Esistono vari tipi di giornalismo dei dati e Mark Coddington (2015) ha sviluppato una classificazione per l'uso dei dati nel giornalismo. Una di queste si chiama CAR (computer-assisted reporting), ovvero il predecessore del giornalismo dei dati e consiste nelle prime applicazioni di tecniche statistiche e visive all'interno dell'attività giornalistica. La seconda tecnica è il giornalismo dei dati in senso proprio; si tratta del termine più usato oggigiorno, ma anche del prototipo della combinazione dei dati e del giornalismo. La terza tipologia è costituita dal giornalismo computazionale, facendo riferimento al modello algoritmico o di Buzzfeed/Vox, il quale si basa su algoritmi che determinano tendenze o l'importanza di determinate vi- 
cende per un certo pubblico e che poi forniscono articoli rilevanti. Si riferisce tuttavia anche, in senso più ampio, alle "pratiche o ai servizi costruiti intorno agli strumenti informatici a servizio di scopi giornalistici" (Coddington 2015: 336).

Il giornalismo dei dati si basa sulla ricerca dei dati (più che delle notizie), l'accento viene quindi posto sul dove e sul quanto reperire dati precisi e affidabili. Spesso il giornalismo dei dati utilizza le statistiche ufficiali o le banche dati fornite da controparti affidabili. Per esempio, Twitter ha donato una banca dati di due milioni di tweet sulle sommosse avvenute a Londra nel 2012 al The Guardian, il quale ha poi realizzato uno degli articoli più memorabili del giornalismo dei dati. In secondo luogo, il giornalismo dei dati richiede, a causa della complessità dell'analisi dei dati e delle competenze richieste, una collaborazione tecnica. Serve inevitabilmente un'equipe in cui "il tecnico incontra il bravo scrittore" (Gray et al. 2012), dove gli studiosi dei dati, i giornalisti ed i grafici si incontrano per produrre un giornalismo all'avanguardia. Infine, il giornalismo dei dati è o può essere open source: in un caso d'avanguardia sulle spese dei deputati, The Guardian si è basato sugli utenti i quali hanno passato al setaccio la parte di dati loro assegnata e hanno poi riferito al giornalista-coordinatore. Ciò ha permesso anzitutto la partecipazione diretta dei lettori nella realizzazione dell'articolo e, in secondo luogo, ha reso il processo di analisi di vaste quantità di informazioni più gestibile.

Gli articoli del giornalismo dei dati tendono ad essere molto ricchi e densi in termini di informazioni. Includono parti testuali, visive e interattive/personalizzate. Occasionalmente l'informazione viene presentata sotto forma di infografiche, le quali semplificano e riducono la complessità sintetizzando i punti chiave. Gli articoli del giornalismo dei dati tendono a diventare storie complesse e composte da più parti che aiutano a trasmettere la complessità del mondo. Vi si trovano collegamenti inaspettati, sviluppi e collegamenti che rivelano dinamiche sottostanti, occasionalmente di modi di fare errati come in Lux Leaks e che contribuiscono ad una comprensione del mondo più profonda. In termini di presentazioni, il giornalismo dei dati segna un cambiamento verso forme che impegnano anche la componente visiva le quali consentono ai lettori di apprendere visualmente i vari collegamenti. Siccome nel giornalismo dei dati l'accento è posto sui dati e su interpretazioni e analisi basate sui fatti a differenza delle forme di giornalismo soggettive e di opinione, viene spesso considerato una forma di giornalismo più accurata e meno di parte.

D'altro canto, il giornalismo dei dati è una forma giornalistica che richiede un lavoro intenso e che potrebbe non essere sufficientemente sostenuta da strutture mediatiche che già subiscono forti pressioni. Ma forse il 
problema maggiore consiste nel fatto che il giornalismo dei dati dà l'impressione di fattualità. In ogni modo, i dati non sono e non rappresentano la "verità". Questa focalizzazione sui dati oscura la politica che ne costituisce la base, le potenziali faziosità nella raccolta, analisi e addirittura nella pubblicazione dei dati. Pielke (2015), per esempio, analizza il modo in cui il giornalismo dei dati ha sbagliato nelle elezioni nel Regno Unito del 2015. Nate Silver di FiveThirtyEight, una start-up giornalistica basata sui dati, ha riferito con insistenza che nessun singolo partito avrebbe ottenuto la maggioranza in Parlamento alle elezioni, dando adito ad ulteriori articoli sul tema. Pielke riporta che Sylvation, un opinionista britannico, aveva realizzato una previsione più precisa, ma non l'aveva pubblicata a causa della dominanza dei sondaggi che non intravedevano una maggioranza parlamentare ed a causa delle pressioni esercitate da media molto noti quali $\mathrm{Fi}$ veThirtyEight. Siccome, in fondo, i dati non mentono mai, ciò lascia poco spazio per esprimere un dissenso nei confronti di essi e delle loro interpretazioni. Secondo Pielke (2015, non numerato) il ruolo dei giornalisti dei dati "non dovrebbe consistere in una limitazione, intenzionale o no a causa del peso della propria influenza, del dibattito pubblico, ma nell'aprire lo stesso. Ciò significa guardare dietro ai numeri e dentro a tutta la confusione della politica. La sapienza che difetta di prove e dati precisi è impoverita. Ma anche i dati e le prove che difettano di un contenuto politico sono impoveriti”. In breve, nel giornalismo dei dati gli articoli valgono tanto quanto i dati ma, allo stesso tempo, gli articoli valgono tanto quanto l'interpretazione che i giornalisti danno ai dati.

Un ulteriore problema che riguarda il giornalismo dei dati consiste nel fatto che, nonostante una delle conquiste per il pubblico dei nuovi media consista nella rimozione della distanza tra i giornalisti ed il pubblico, l'affidamento a ricercatori ed esperti di dati introduce nuove barriere. Ciò crea tensione tra gli studiosi dei dati che conoscono i dati ma non il giornalismo ed i giornalisti, i quali non conoscono i dati né la loro produzione, ma che hanno il compito di interpretarli. Infine, sono poche le prove che abbiamo a disposizione sul come queste nuove forme di giornalismo vengano recepite dagli spettatori/dal pubblico. Rappresentano meramente un modo semplice di narrare vicende complesse o si ritiene che esse rendano poco chiaro e complichino senza necessità le vicende?

Per concludere, la sfida del giornalismo dei dati comprende la riconciliazione della lettura dei dati e del senso di importanza e di analisi tipico del giornalismo, dando la priorità a una collaborazione a pari livello più che a sostituire i giornalisti con gli esperti dei dati. Inoltre, la sfida che qui si pone non consiste nell'assumere che i dati equivalgano sempre alla verità, bensì nello sviluppare nuovi modi creativi di fare ricerche nel mondo 
dei dati. In senso più ampio, tutte le nuove forme di giornalismo offrono contributi positivi, ma hanno, allo stesso tempo, anche dei lati negativi. Forse la chiave consiste nella coesistenza di tutte queste forme. Alla domanda se tutte queste nuove forme siano positive per il giornalismo, la risposta deve essere senz'altro affermativa. Ciò in quanto mentre per il tipo ideale di giornalismo il pubblico è informato, razionale, critico e sempre interessato alle notizie, la realtà è più disordinata. Le diverse forme di giornalismo si rivolgono a pubblici diversi o al medesimo pubblico in modi diversi. Gli offrono una rinnovata tensione tra la forma ed il contenuto e, di conseguenza, nuovi significati. Forse non rimpiazzeranno le forme classiche della piramide inversa o gli articoli di opinione, ma offriranno nuove esperienze ai lettori, richiamando, di conseguenza, nuovi lettori nel processo e mettendo a fuoco tratti finora inesplorati della realtà.

\subsection{Lettori, utenti, pubblico, spettatori}

Un discorso sui lettori completa il circuito della comunicazione nel giornalismo. I termini sopracitati riflettono la complessa rete di rapporti instauratasi tra il giornalismo ed il suo pubblico. Nonostante esistano intensi dibattitti su ognuno di questi termini, in questa sede vengono usati come sinonimi per svolgere alcune riflessioni sui rapporti passati e presenti (lettori e utenti), sul ruolo pubblico del giornalismo (pubblico) e sui diversi modi in cui i diversi media - specialmente le emittenti televisive - considerano i loro "spettatori". Una delle questioni e delle sfide centrali che si presentano al giornalismo è quella della scomparsa dei lettori. Il ben documentato calo della diffusione dei giornali insieme ad una forte competizione per accaparrarsi l'attenzione nel nuovo ambiente mediatico contribuisce ad una crescente preoccupazione in merito alla sparizione del pubblico del giornalismo. Ma è davvero così? Oppure questo pubblico ha semplicemente cambiato le proprie abitudini di consumo? Questa tematica verrà affrontata nella prima parte del presente capitolo. In secondo luogo, è profondamente mutata la natura dell'identità dei consumatori del giornalismo, tradizionalmente intesi come lettori passivi o pubblico; teorici come Bruns (2006) li hanno definiti "produsers" ("produttori-utenti"). Questo cambiamento verrà trattato insieme al mutamento dei contenuti generati dagli utenti nella seconda parte del presente capitolo. Infine, il capitolo si concluderà con una considerazione sul modo in cui i giornalisti stessi percepiscono il loro pubblico e su quanto questo fattore possa portare a cambiamenti più ampi nel giornalismo. 


\subsubsection{Il calo dei lettori e il cambio di abitudini}

Negli anni recenti si è assistito ad un calo ben documentato della diffusione dei quotidiani. Per esempio, Pew negli Stati Uniti ed i sondaggi di ADC nel Regno Unito evidenziano cali di circolazione nel mercato dei quotidiani, e tale fenomeno è riscontrabile in quasi tutta Europa (Pew Research Center 2015; Greenslade 2014). Significa che ai lettori non interessa più l'informazione? Un'analisi più approfondita rivela due tendenze prevalenti: di fatto, meno persone sono "consumatori" di notizie, i giovani tendono sempre meno a leggere la carta stampata, ma anche le piattaforme dell'informazione ed i media sono cambiate, ed i giovani prediligono internet alla televisione o ai giornali. Il grafico 3.1 mostra che i c.d. "millennials" trascorrono meno tempo per seguire l'informazione, mentre sia i "millennial" che la "generazione $X$ " preferisce reperire le notizie su internet (rispettivamente il 43\% e il 49\%). In ogni modo, da entrambe le categorie non arrivano buone notizie per il giornalismo: vi è una "base di clienti" in calo e le nuove piattaforme ed i nuovi modelli di distribuzione, specialmente i social media, indeboliscono i modelli commerciali predominanti, come già visto precedentemente (Generazione silenziosa: 1925-1945, Baby boomer: 1946-1964, Generazione X: 1965-1980, Millennial: 1981-1997 (Fry 2015)).

Grafico 3.1 - Differenze nel consumo di notizie

\section{Scarti generazionali}

\section{nel tempo trascorso a seguire le notizie}

Numero medio di numero di minuti trascorsi al giorno a seguire le notizie, per fasce d'età"

Generazione silenziosa

2004

2006

2008

2010

2012

\section{Baby} boomer

75

71

72

79

77
Generazione X

63

63

64

71

66
Millennial

43

45

43

45

46

* il giorno prima del sondaggio.

Fonte: Pew Research Center 2015.

Sorgono qui due domande. I giovani lettori si interesseranno di più per l'informazione una volta cresciuti? Oppure il loro interesse aumenterà se le 
piattaforme e/o i contenuti dell'informazione terranno conto dei loro gusti? Se quest'ultima ipotesi corrisponde a verità, ciò fa pressione sul giornalismo tradizionale, che ancor oggi si svolge su carta stampata, affinché scovi nuovi modi per richiamare il pubblico. Buzzfeed, Vice e Gawker rappresentano esempi del tipo di informazione che sembra piacere ai "Millennials" se guardiamo al numero di condivisioni e di like che questi tipi di informazione ottengono. Reuters ha ritenuto necessario introdurre una nuova categoria di notizie - le notizie "divertenti/strane" ("fun/weird news") - per offrire questo tipo di contenuti.

Comprendere i gusti del pubblico presuppone la comprensione del cambiamento delle abitudini di consumo dell'informazione. Uno dei mutamenti principali accaduti negli ultimi anni riguarda la distribuzione sociale dell'informazione. Detto in parole semplici, sempre più lettori si affidano ai social media per accedere all'informazione. Come dimostra il report sui media dell'informazione digitali di Reuters del 2015, vi è un costante aumento del numero di persone che utilizzano i social media per accedere all'informazione.

\section{Grafico 3.1 - Accesso all'informazione tramite social media}

\section{PERCENTUALE DI PERSONE CHE UTILIZZANO I SOCIAL NETWORK PER ACCEDERE ALL'INFORMAZIONE, PERIODO 2013-2015}

Per Paesi scelti

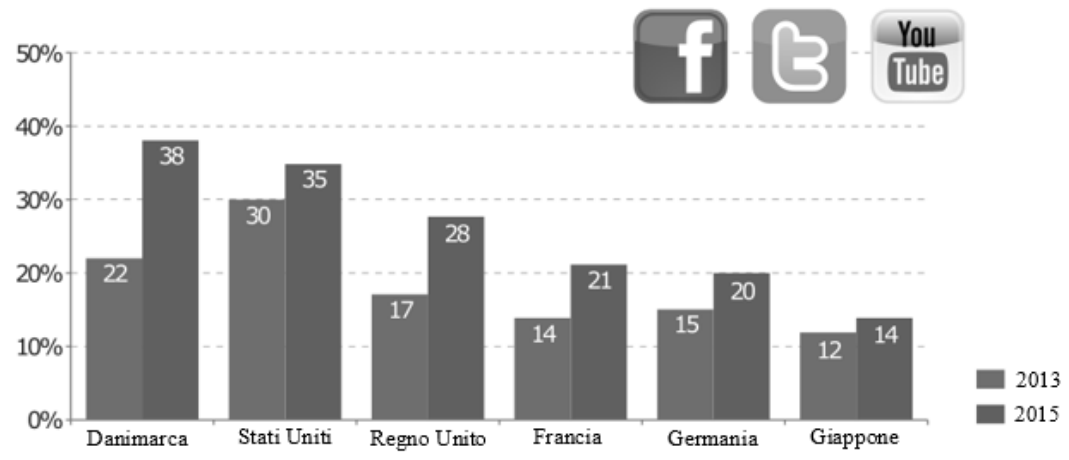

Fonte: Reuters Institute 2015.

All'interno di questo contesto sono emersi due tipi di distribuzione: uno si basa sui nuovi c.d. "infomediari" (Rebillard e Smyrnaios, 2010), social media che fungono da nuovi mediatori dell'informazioni, mentre l'altro riguarda una distribuzione sociale basata sui network degli utenti stessi. Le 
persone tendono a leggere ciò che gli altri hanno condiviso sulla propria pagina e condividono a loro volta, commentano o mettono "mi piace" a detti contenuti. In ogni modo, la condivisione sociale presuppone che l'utente abbia rinvenuto una notizia sulla propria bacheca. Ma ciò che vediamo dipende sempre più da come i social media ordinano la nostra bacheca, e ciò dipende da algoritmi che ordinano e selezionano le informazioni in modi specifici.

La questione diventa cruciale nel caso di Facebook che si sta profilando come distributore sociale dell'informazione. Gli algoritmi di Facebook, OpenGraph, EdgeRank e GraphRank, si basano su dati generati dalla partecipazione passiva delle persone (Bücher 2012). Ma non è sufficiente raccogliere dati per identificare le tendenze e gli andamenti. Gli algoritmi e i protocolli di Facebook sono orientati verso il futuro e lo anticipano. Essendo l'anticipazione già contenuta negli algoritmi, essi tendono a diventare una profezia che si avvera da sola: scrivono il futuro nel modo in cui lo anticipano. In altre parole, se i giovani utenti di Facebook mettono "mi piace" e condividono solo notizie della pagina di Buzzfeed, nella loro bacheca appariranno soltanto notizie del genere. Così facendo, Facebook riduce e previene azioni alternative e future, diminuisce l'imprevedibilità delle notizie e riproduce ciò che è noto come filtro bolla (Pariser 2012). Il filtrobolla si riferisce al tipo di ambiente che spesso si crea nei social media, ove le persone seguono soltanto le persone simili a sé stesse, con opinioni, idee e antipatie simili, e difficilmente entreranno in contatto con qualcos'altro. Le bacheche iper-personalizzate, il risultato di una combinazione degli algoritmi di Facebook e la ben nota tendenza dei network verso l'omofilia l'associazione con soggetti simili - costituiscono un filtro-bolla il quale scarta qualsiasi tipo di informazione "dissenziente".

È molto curioso che Eli Pariser, l'autore di The filter bubble: what the internet is hiding from you abbia creato Upworthy, un mezzo con il quale far circolare informazioni rilevanti attraverso i filtri-bolla delle persone convincendo loro a condividerle. Upworthy è ora considerato un esempio di giornalismo c.d. "clickbait". Ciò riassume la sfida che si pone per il giornalismo: rimanere rilevanti, ottenere l'impegno, essere condiviso e ricevere "mi piace" affinché vengano raggiunte sempre più persone, però senza scadere nel "clickbait" oppure offrendo notizie divertenti/strane.

Una caratteristica nonchè risultato dei social network consiste nel fatto che essi sembrano sostituire o replicare le decisioni editoriali. Tuttavia, queste non vengono più prese sulla base dei valori di stampa o della rilevanza di cronaca, bensì sulla base dei modelli, dei "mi piace" e delle condivisioni precedenti dei lettori. In ogni modo, ciò potrebbe portare alla liquidazione del significato, in quanto la viralità o il grado di condivisione di- 
ventano il criterio principale. In altre parole, il valore e il significato del contenuto non vengono presi in considerazione: il significato è sostituito dalla popolarità (Siapera 2013). La popolarità, che una volta era soltanto un segno di importanza, è ora diventata una finalità in sé. Ma non necessariamente la notizia più importante o più significativa è quella più condivisa. In termini più ampi, questi modelli che cambiano denotano un cambiamento nel potere dagli editori tradizionali agli "infomediari" dei social network e la sostituzione delle decisioni editoriali umane per mezzo di ordini effettuati e priorità stabilite da algoritmi automatici.

\subsubsection{Gli utenti come "produser"}

La sfida che i nuovi "infomediari” rappresentano per il giornalismo e per l'editoria dell'informazione è accompagnata da una sfida ulteriore, questa volta proveniente non già dai modelli di consumo dei lettori, bensì dalle loro capacità di produrre notizie. Il termine inglese "produsage", coniato da Axel Bruns nel 2007, si riferisce alla prassi ibrida emersa negli ambienti condivisi, ovvero, secondo l'autore, una prassi dietro la produzione. L'idea chiave consiste nel fatto che "ognuno possa creare", gli utenti divengono produttori di contenuti, mentre i risultati non sono più prodotti diversi si tratta di artefatti contemporanei di un processo continuo. L'utilizzo e la produzione sono sempre più inestricabilmente intrecciati e non si rilevano più rigide distinzioni tra produttori, distributori e consumatori. Questo nuovo termine è stato coniato per sottolineare la fine di un modello di consumo passivo e l'inizio di una nuova era nelle relazioni tra il pubblico e i media.

Cosa implica tutto ciò per il giornalismo ed il suo pubblico? Molti contenuti prodotti dagli utenti non corrispondono esattamente agli standard giornalistici accettati o accettabili. Nondimeno, ignorare tali contenuti ha un costo potenzialmente alto per il giornalismo, il quale potrebbe essere messo da parte. La partecipazione del pubblico è durevole e ciò significa che il giornalismo deve adattarsi. Ma non si tratta di un processo facile. La partecipazione degli utenti è spesso un anatema per i giornalisti. In uno studio sugli editori del Regno Unito, Hermida e Thurman (2008) hanno scoperto che essi si sentivano forzati a includere i contenuti generati dagli utenti più che a lasciarli da parte. In ogni modo, essi devono affrontare le tensioni che emergono dall'esigenza di controllare il proprio marchio, l'esigenza di controllare il dibattito ed i costi di un tale controllo. Complessivamente, sembra che i giornalisti vogliano ancora conservare la propria funzione di guardiani e che i contenuti degli utenti potrebbero indebolir- 
la. In ogni modo, le abitudini potrebbero cambiare. Lewis si esprime a favore di un giornalismo più flessibile, aperto, caratterizzato dalla "disponibilità di considerare maggiormente il pubblico a pari livello, di apprezzarne i contributi e di trovare scopi normativi nella trasparenza e nella partecipazione" (Lewis 2012: 851).

Allo stesso tempo, data la natura dell'informazione, che è imprevedibile, (quasi) casuale e sempre più globalizzata, sempre più media ritengono di fare affidamento ai contenuti generati dagli utenti. Ciò comporta sfide importanti di natura pratica, etica e giuridica. È importante fare presente che i contenuti generati dagli utenti costituiscono parte integrante dell'informazione di oggi, dalle immagini/dai video alle notizie in tempo reale ai video atroci/di propaganda girati dall'ISIS. Wardle et al. (2014) hanno svolto un'accurata ricerca sui contributi generati dagli utenti nelle redazioni. I principali risultati di tale studio comprendono il fatto che tali contributi vengono utilizzati specialmente quando non sono disponibili altre fonti. Una delle tematiche centrali in merito ai contenuti generati dagli utenti è legata alle etichette, in quanto il $72 \%$ di tali contenuti non viene contraddistinto come tale. Secondo Wardle et al. soltanto il 16\% dei contenuti generati dagli utenti trasmessi in televisione includono il riferimento alla persona che ha realizzato il filmato.

Wardle et al. (2014) chiedono lo sviluppo di procedure più sistematiche ad uso dei redattori di notizie d'attualità affinché essi siano in grado di utilizzare in modo credibile e di riconoscere i contributi generati dagli utenti. Un'importante questione giuridica che inevitabilmente sorgerà riguarda l'autorizzazione di tali contenuti. Non è improbabile che vengano proposte delle class action contro le organizzazioni degli utenti per aver usato tali contenuti cosicché esse dovranno essere preparate a ciò. Inoltre, esistono alcune implicazioni etiche e, nello specifico, il pericolo che i cittadini siano esposti a pericoli nella loro richiesta di ottenere le foto/i video che probabilmente verranno pubblicati o trasmessi. Wardle et al. riferiscono che The Guardian ha cambiato i termini utilizzati: invece di "inviaci" le tue immagini si legge ora "condividile con noi" quale mezzo minimo per evitare di esporre i lettori a pericoli. Vi è poi un'altra questione etica e psicologica in gioco che riguarda i giornalisti esposti a video amatoriali che mostrano disastri o atrocità. Le imprese mediatiche devono prendere sul serio il benessere psicologico dei loro dipendenti ed evitare di esporli inutilmente a traumi. 


\subsubsection{Ripensare i rapporti}

I cambiamenti descritti precedentemente stanno lentamente, ma sicuramente tenendo vivo il processo di produzione dell'informazione. Mentre nell'era della carta stampata e del giornalismo radiotelevisivo i giornalisti conoscevano poco i loro lettori/il loro pubblico se non tramite le lettere che pervenivano alla redazione, oggi si conoscono nel dettaglio le abitudini, i commenti, i "mi piace" e le abnegazioni dei lettori. Inoltre, ogni caporedattore ha ora a propria disposizione stime dettagliate sulle abitudini del proprio pubblico. Fino a quanto ciò influisce sulle decisioni editoriali? E fino a quanto ciò dovrebbe influirvi?

La ricerca ci dice che tali stime stanno ottenendo un'importanza crescente per l'editoria dell'informazione. Anderson (2011) ha rilevato che ai giornalisti vengono fatte presenti le stime sulla diffusione delle notizie e che viene richiesto loro di scrivere articoli che soddisfino i gusti del pubblico. Similmente, Tien $\mathrm{Vu}$ (2013) ha constatato che i giornalisti tendono a privilegiare gli articoli con alti tassi di diffusione. Boczkowski e Mitchelstein (2013) hanno scoperto una lacuna consistente tra ciò a cui i giornalisti/i caporedattori danno la priorità e gli articoli su cui clicca il pubblico.

Posto l'accento sull'impegno del pubblico, l'onere grava ora sui giornalisti affinché essi costruiscano e mantengano vivo un rapporto con i loro lettori. Creare una comunità, il che richiede un lavoro affettivo, potrebbe essere uno strumento potenziale per creare e mantenere vivi i rapporti. Come visto in precedenza, il lavoro affettivo si inserisce in quella parte del lavoro giornalistico che riguarda la socializzazione con i lettori. Non si tratta di giornalismo in senso stretto, ma di un aspetto sempre più necessario dello stesso. La nozione di giornalismo reciproco (Lewis et al. 2014) si basa sull'idea di inserire nel giornalismo il concetto di reciprocità con il pubblico. In altre parole, il giornalismo non consiste più solo nello scrivere e nel pubblicare un articolo, ma anche nell'analizzarlo insieme ai lettori, coinvolgendo le loro domande ed i loro commenti, riflettendo sulle loro critiche, ecc. In ogni modo, tale prassi è fondamentalmente ambigua in quanto estrae la socialità e lo scambio sociale da un contesto segnato da relazioni sociali e le rende parti di un'attività professionale. Sapersi comportare bene è un conto, rendere ciò il proprio principale argomento di vendita è un altro. Nello studio sui giornalisti su Twitter redatto da Siapera e Iliadi (2015), chi ha risposto era ostinatamente convinto che l'autenticità è cruciale: bisogna comprendere i lettori nella loro personalità, e le finzioni verranno scoperte e rese pubbliche. In ogni modo, quanti sono i giornalisti che possono fare ciò liberamente se lavorano per un marchio? Il giornali- 
smo deve affrontare queste tensioni per essere in grado di ricostruire il proprio rapporto con il pubblico.

\section{CONCLUSIONI}

Il presente saggio si è aperto con una considerazione sul processo della comunicazione inteso come un circuito che comprende la produzione, il contenuto ed il consumo od uso. Ma, nel corso della trattazione, abbiamo approfondito alcuni casi che complicano tali processi e che mettono in luce l'esigenza di modificare tale circuito tenendo in considerazione i cambiamenti recenti. Il grafico 4.1 costituisce un tentativo del genere.

\section{Grafico 4.1 - Il nuovo circuito del giornalismo digitale}

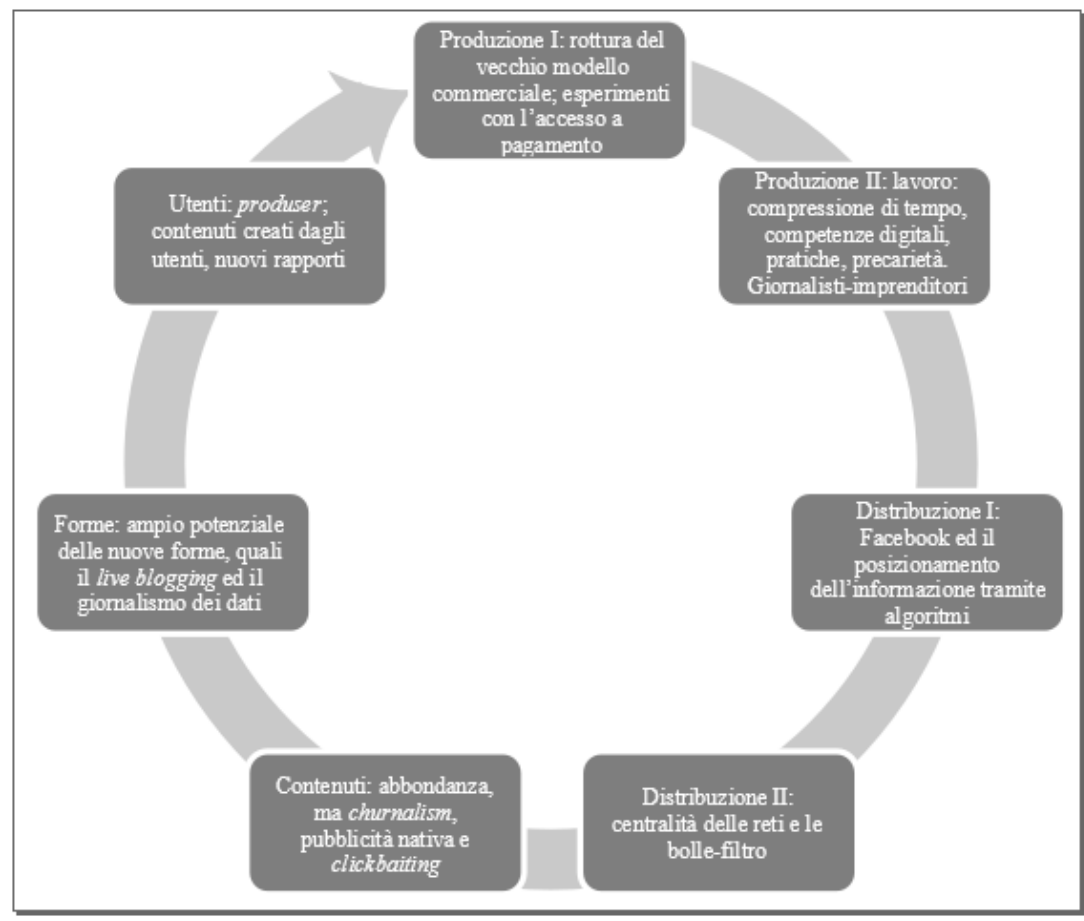

Per ricapitolare le principali tesi sopra esposte, in termini di produzione la sfida principale per il giornalismo consiste nel trovare una nuova fonte di guadagno che non riproponga le problematiche dei modelli passati. La 
commodificazione dell'informazione, insieme alla commodificazione del pubblico, rappresentavano già aspetti problematici del giornalismo inteso come pubblico servizio. L'ascesa dell'imprenditoria non risolve i problemi, bensì si limita a trasferirli dagli editori ai giornalisti stessi. La precarietà e l'incertezza comportano indubbiamente problemi seri ai giornalisti, rendendoli più vulnerabili allo sfruttamento e più inclini a compromessi che potrebbero finire per compromettere il giornalismo in senso più lato.

Seguendo questa analisi e ritenendo che tali fenomeni impattano su tutti i livelli, riteniamo necessario includere un processo che era stato ritenuto sotto il controllo della produzione e di imprese che producono l'informazione - la distribuzione. Non è più così: la distribuzione digitale è saldamente nelle mani di social network quali Facebook o Twitter o motori di ricerca quali Google. Inoltre, la distribuzione ha acquisito importanza singolare nell'era dei social media a causa della sovrabbondanza di contenuti e dell'esigenza di riordinare la visibilità dei media (cfr. Thompson 2005). Tuttavia, l'ordinamento algoritmico del dominio della visibilità è pieno di problemi. Le decisioni algoritmiche dipendono dal comportamento precedente degli utenti e difficilmente esporranno questi ultimi a contenuti nuovi o inaspettati, contribuendo a creare ciò che Pariser ha definito un "filtro-bolla". Secondo una distribuzione algoritmica dell'informazione le notizie importanti ma impopolari potrebbero finire per essere rimosse. La sfida, per il giornalismo, consiste nel reclamare la propria autorità smarrita nell'imporre l'agenda mediatica nonché il proprio diritto di prendere decisioni editoriali sulla base della rilevanza di cronaca anziché sul numero dei "mi piace", delle condivisioni e dei commenti.

Tale contesto di produzione e di distribuzione ha avuto un chiaro impatto sul contenuto. L'ascesa del c.d. "churnalism" (il copia e incolla del medesimo contenuto), del c.d. "clickbait" (l'indurre i lettori a fare un click) e la "pubblicità nativa" (i mezzi quasi ingannevoli con i quali si spingono $\mathrm{i}$ lettori a leggere gli annunci pubblicitari) hanno pressoché schiacciato il potenziale di internet di produrre un giornalismo impegnato e veramente accattivante. Chiaramente, la sfida qui rilevante consiste nel rigenerare tale potenziale e nell'investire nell'arricchimento reciproco del giornalismo con le "affordances" tecnologiche dei nuovi media. In questo potenziale, così come nella sua ambiguità, ci si imbatte chiaramente nel considerare le nuove forme del giornalismo, i nuovi modi in cui ordinare ed organizzare i contenuti. Altri modi, non analizzati in questa sede includono il giornalismo mobile e il c.d. giornalismo "immersivo" ("immersive journalism"). Il giornalismo deve continuare a sperimentare tali forme, unendo il meglio di sé stesso al meglio dei nuovi media, La sfida consiste nel trovare spazio per una tale sperimentazione produttiva. 
Il ruolo degli utenti è diventato più importante nell'ecosistema mediatico. Mentre, nelle teorie precedenti, l'attività principale degli utenti consisteva nell'interpretazione dei messaggi mediatici, ora essi possono reagire in forma attiva a tali messaggi, modificandoli, commentandoli e addirittura producendone di nuovi. Tutto ciò comporta un considerevole mutamento di potere, ma non significa la mancanza dell'esigenza del giornalismo e del suo valore aggiunto. In ogni modo, evidenza chiaramente l'esigenza di tenere conto dei lettori ad ogni livello. Dal punto di vista negativo, ciò implica l'ossessione incessante per le statistiche, il che potrebbe poi avere una ricaduta sulla produzione del giornalismo, portando ad un giornalismo più innocuo; ma forse meno incisivo e meno utile dal punto di vista sociale. Tuttavia, vi sono anche degli aspetti positivo. Tale fenomeno potrebbe difatti portare alla nascita di una giornalismo reciproco in cui i giornalisti creano una specie di comunità. Ma all'interno di un contesto in cui il giornalismo è considerato una merce e i giornalisti dipendenti, tutto ciò è profondamente ambiguo. Allo stesso tempo, i giornalisti devono trovare nuove strade per riconciliare i contenuti generati dagli utenti con i valori e le concezioni del giornalismo. La sfida consiste nel relazionarsi con i lettori/utenti e con i loro contenuti in modo significativo che dia un valore aggiunto al giornalismo.

Tutti questi fenomeni in quale posizione pongono il giornalismo? La sua posizione è precaria e paradossale: mentre è chiaro che la necessità e il valore del giornalismo rimangono invariati, e forse sono oggi più importanti che mai, esso affronta sfide complesse senza precedenti, che provengono da tutte le parti: dagli editori, dalle condizioni di lavoro, dai social media e dagli utenti. Per nessuna di queste sfide vi sono risposte facili. In ogni modo, rimangono alcuni punti da tenere in considerazione nell'affrontare alcune di queste sfide.

Posti l'importanza e il valore del giornalismo, qui è in gioco la risocializzazione dello stesso: il ritorno del giornalismo alla società che intende servire. La risocializzazione del giornalismo ne richiede la rimozione dalla propria posizione sul mercato. In ogni modo, ciò non vuol dire necessariamente un giornalismo sovvenzionato dallo Stato con tutti gli oneri che ciò comporterebbe. Dovremmo, invece, prendere in considerazione forme diverse di organizzazione economico-sociale quali le cooperative e le forme che non hanno scopo di lucro. Vi sono dei vantaggi considerevoli, in quanto si consente al giornalismo di operare in una modalità indipendente, ma sostenibile, reinvestendo gli utili nella cooperativa stessa e per pagare lo stipendio ai giornalisti. In un tale contesto, in quanto i giornalisti lavorerebbero per il giornalismo e non per il lucro soggettivo di qualcun altro, l'aspetto sociale legato alla creazione di una comunità, il "biopouvoir" coin- 
volto nel lavoro affettivo dei giornalisti, la produzione delle soggettività e della comunità possono essere librati dalla commodificazione e al giornalismo si consentirebbe di realizzare tutto il suo potenziale.

In secundis, per quanto riguarda la diffusione dell'informazione, i giornalisti e gli utenti devono entrambi esercitare pressioni su Facebook e sugli altri social media affinché offrano più scelte nell'ordinamento delle bacheche, magari sviluppando un algoritmo "giornalistico" che adoperi il criterio giornalistico della rilevanza di cronaca e i valori dell'informazione. Ciò può coesistere insieme ad altri algoritmi basati sul numero delle condivisioni sull'impegno, il criterio cronologico, ecc. Facebook sta già svolgendo alcuni esperimenti con partner mediatici sui c.d. "instant articles", tuttavia si tratta di un'altra piattaforma per l'informazione che non risolve la questione della divulgazione e della visibilità (Reckhow 2015). Inoltre, potrebbe altresì privilegiare i grandi nomi a discapito dei distributori di informazione minore e delle iniziative giornalistico. Finché Facebook e gli altri social network non indirizzeranno direttamente la distribuzione algoritmica dell'informazione, la popolarità prevarrà sempre sull'importanza.

Dato che queste sfide si intensificheranno e acquisteranno sempre maggiore importanza, è probabile che nessuna soluzione emergerà in forma organica dalla prassi dei vari attori stessi. In ogni modo, in mancanza di una qualsiasi forma di guida sociale nel ridirigere il giornalismo direttamente verso la società, è analogamente probabile che tali situazioni possano, in ultima analisi, distruggere il giornalismo inteso come pubblico servizio.

\section{BIBLIOGRAFIA}

Adorno T. (2013 [versione originale 1970]), Aesthetic Theory, Bloomsbury.

Anderson C. W., Bell E. e Shirky C. (2012), "Post-industrial journalism: adapting to the present: a report", Columbia Journalism School.

Anderson K. (2011), "Live blogging evolved: context and curation not just collection", disponibile su: http://charman-anderson.com/2011/02/23/live-blogging-ev olved-context-and-curation-not-just-collection/, consultato il 20 luglio 2015.

Arrese Á. (2015), From Gratis to Paywalls: A brief history of a retro-innovation in the press's business. Journalism Studies (2015), pp. 1-17.

Beckett C. (2010), "The Value of Networked Journalism." Polis Working Paper, Department of Media and Communications, London School of Economics \& Political Science.www2.lse.ac.uk/media@lse/POLIS/documents/Polis\%20papers /ValueofNetworkedJournalism.pdf.

Bishton D. (2001), "From DT to ED", in The Daily Telegraph, 1 gennaio 2001, disponibile su: www.telegraph.co.uk/news/1471964/From-ET-to-TD.html, consultato il 20 luglio 2015. 
Blom J. N. e Hansen K. R. (2015), "Click bait: forward-reference as lure in online news headlines", Journal of Pragmatics, vol. 76, 87-100.

Boczkowski P. J. \& Mitchelstein E. (2013), The news gap: When the information preferences of the media and the public diverge, MIT press.

Branch J. (2012), "Snowfall: the avalanche of Tunnel Creek", The New York Times, disponibile su www.nytimes.com/projects/2012/snow-fall/\#/?part=tunnel-creek, consultato il 20 luglio 2015.

Brick S. (2012), "There are downsides to looking this pretty: why women hate me for being beautiful", in MailOnline, 3 aprile 2012, disponibile su: www.dailymai l.co.uk/femail/article-2124246/Samantha-Brick-downsides-looking-pretty-Why-w omenhate-beautiful.html\#ixzz3cqwkAIis, consultato il 20 luglio 2015.

Bruns A. (2006), “Towards Produsage: Futures for User-Led Content Production.” In Sudweeks F., Hrachovec H. ed Ess C. (a cura di), Proceedings Cultural Attitudes towards Communication and Technology 2006, pp. 275-284, Tartu, Estonia.

Bruns A. (2012), "Journalists and Twitter: how Australian news organisations adapt to a new medium”, Media International Australia incorporating Culture and Policy, pp. 97-107.

Bucher T. (2012), "Programmed sociality: a software studies perspective on social networking sites", Doctoral dissertation, University of Oslo.

Byrne L. (2014), "Storyful accounts reveal losses of $€ 3 m$ prior to News Corp sale", Irish Independent, 12 gennaio 2014, disponibile su: www.independent.ie/irish-ne ws/storyful-accounts-reveal-losses-of-3m-prior-to-news-corp-sale-29908344.html, consultato il 20 luglio 2015.

Castells M. (2000), "Materials for an exploratory theory of the network society", The British Journal of Sociology, 51(1), 5-24.

Chowdhry A. (2014), "Facebook is going to suppress 'click-bait' articles", disponibile su www.forbes.com/sites/amitchowdhry/2014/08/26/facebook-is-going-to-su ppressclick-bait-articles/, consultato il 16 luglio 2015.

Christensen C. M. (1997), “The innovator's dilemma: the revolutionary book that will change the way you do business", Collins Business Essentials.

Coddington M. (2015), “Clarifying journalism's quantitative turn: a typology for evaluating data journalism, computational journalism, and computer-assisted reporting", Digital Journalism, Vol. 3, n. 3, pp. 331-348.

Curran J. e Seaton J. (2003), Power without responsibility: press, broadcasting and the internet in Britain, Routledge.

Cushion S. e Lewis J (a cura di) (2010), The rise of 24-hour news television: global perspectives, Peter Lang Publishing Group.

Deuze M. e Fortunati L. (2010), "A typical newswork, atypical media management”, in Deuze M. (a cura di), Managing Media Work, Sage, London.

Deuze M. (2009), “The people formerly known as the employers”, Journalism, Vol. 10, n. 3, p. 315.

Dick M. (2011), "Search engine optimisation in UK news production", Journalism Practice, Vol. 5, n. 4, pp. 462-477. 
EMarketer (2014), "Can Native Advertisers Avoid Confusing UK Consumers?", disponibile su www.emarketer.com/Article/Native-Advertisers-Avoid-ConfusingUKConsumers/1011719, consultato il 20 luglio 2015.

Fry R. (2015), This Year Millennials will overtake Baby Boomers, Pew Research Centre, www.pewresearch.org/fact-tank/2015/01/16/this-year-millennials-willovertak e-baby-boomers/

García Avilés J. A., León B., Sanders K. e Harrison J. (2004), "Journalists at digital television newsrooms in Britain and Spain: workflow and multi-skilling in a competitive environment", Journalism Studies, vol. 5, n. 1, pp. 87-100.

Gibson J. J. (2014 [1979]), The ecological approach to visual perception: classic edition, Psychology Press.

Gillmor D. (2006), We the media: Grassroots journalism by the people, for the people, O'Reilly Media, Inc.

Golding P. \& Murdock G. (1991), "Culture, communications and political economy", in Curran J. and M. Gurevitch (a cura di), Mass media and society, Edward Arnold, 15-32.

Gray J., Bounegru L. e Chambers L. (a cura di) (2012), The Data Journalism Handbook, http://datajournalismhandbook.org/1.0/en/index.html

Greenfield R. (2012), "What the New York Times's 'Snow Fall' means to online journalism's future, disponibile su www.thewire.com/technology/2012/12/new-y ork-timessnow-fall-feature/60219/, consultato il 20 luglio 2015.

Greenslade R. (2014), "Latest ABCs show newspaper market decline running at 8\% a year", The Guardian, 11 luglio 2014, disponibile su www.theguardian.com/me $\mathrm{dia} /$ greenslade/2014/jul/11/abcs-national-newspapers, consultato il 20 luglio 2015.

Habermas J. (1991), The structural transformation of the public sphere: an inquiry into a category of bourgeois society, MIT Press.

Hall S. (1980, ristampa 1993), "Encoding, decoding", in During S. (a cura di), The cultural studies reader, Routledge, London/New York, pp. 80-103.

Harcup T. (2015), Journalism: principles and practice, Sage.

Hermida A. (2013), "Journalism: reconfiguring journalism research about Twitter, one tweet at a time”, Digital Journalism, vol. 1, n. 3, pp. 295-313.

Hermida A. e Thurman N. (2008), “A clash of cultures: the integration of usergenerated content within professional journalistic frameworks at British newspaper websites”, Journalism Practice, vol. 2, n. 3, pp. 343-356.

Hermida A., Lewis S. C. e Zamith R. (2014), "Sourcing the Arab Spring: a case study of Andy Carvin's sources on Twitter during the Tunisian and Egyptian revolutions", Journal of Computer-Mediated Communication, vol. 19, n. 3, pp. 479-499.

Hutchby I. (2001), “Technologies, texts and affordances”, Sociology, vol. 35, n. 2, pp. 441-456.

Jarvis J. (2009), "The future of journalism is entrepreneurial”, BuzzMachine, 1 novembre 2009, disponibile su: http://buzzmachine.com/2009/11/01/the-futureof-journalism-isentrepreneurial/, consultato il 20 luglio 2015. 
Jarvis J. (2010), “Teaching entrepreneurial journalism”, BuzzMachine, 11 gennaio 2010, disponibile su: http://buzzmachine.com/2010/01/11/teaching-entrepreneur ialjournalism/, consultato il 20 luglio 2015.

Lasorsa D. L., Lewis S. C. e Holton A. E. (2012), "Normalizing Twitter: journalism practice in an emerging communication space", Journalism Studies, vol. 13, n. 1, pp. 19-36.

Leadbeater C. e Miller P. (2004), The Pro-Am revolution: how enthusiasts are changing our economy and society, Demos.

Lee-Wright P., Phillips A. e Witschge, T. (2011), Changing journalism, Routledge.

Lewis S. C. (2012), "The tension between professional control and open participation: journalism and its boundaries", Information, Communication \& Society, Vol. 15, n. 6, pp. 836-866.

Lewis S. C., Holton A. E. e Coddington M. (2014), "Reciprocal journalism: a concept of mutual exchange between journalists and audiences", Journalism Practice, vol. 8, n. 2, pp. 229-241.

Lister M., Dovey J., Giddings S., Grant I. e Kelly K. (2009), New media: A critical introduction, Taylor \& Francis.

Lorenz M. (2012), "Why journalists should use data”, in Gray J., Chambers L. and Bounegru L. (eds), The data journalism handbook, O’Reilly Media, disponibile su: http://datajournalismhandbook.org/1.0/en/index.html, consultato il 20 luglio 2015.

Malaga R. (2008), "Worst practices in search engine optimization”, Communications of the ACM, vol. 51, n. 2, pp. 147-50.

McManus J. H. (1994), Market-Driven Journalism: Let the Citizen Beware? Sage.

Marjoribanks T. (2000), News Corporation, technology and the workplace: global strategies, local change, Cambridge University Press.

Massey B. L. ed Elmore C. J. (2011), "Happier working for themselves? Job satisfaction and women freelance journalists", Journalism Practice, vol. 5, n. 6, pp. 672686.

Meikle G. (2002), Future active: media activism and the Internet, Routledge, Londra/New York.

Murdock G. e Golding P. (1973), "For a political economy of mass communications", Socialist Register, vol. 10, n. 10.

Newman N. (2015), Reuters Institute Digital News Report 2015, disponibile su: https://reutersinstitute.politics.ox.ac.uk/sites/default/files/Reuters\%20Institute\% 20Digital\%20News\%20Report\%202015_Full\%20Report.pdf

Oremus W. (2014), “The first news report on the LA earthquake was written by a robot", Slate, 17 marzo 2014, disponibile su: www.slate.com/blogs/future_tense/ 2014/03/17/quakebot_los_angeles_times_robot_journalist_writes_article_on_la_ earthquake.html, consultato il 20 luglio 2015.

Örnebring H. (2010), “Technology and journalism-as-labour: Historical perspectives", Journalism, 11(1), 57-74. 
Papacharissi Z e Oliveira M (2012), "Affective news and networked publics: the rhythms of news storytelling on \#Egypt”, Journal of Communication, vol. 62, n. 2, pp. 266-282.

Pariser E. (2012), The filter bubble: what the Internet is hiding from you, Penguin, UK. Paulussen S. (2012), “Technology and the transformation of news work: are labor conditions in (online) journalism changing?", in E. Siapera e A. Veglis (a cura di), The handbook of global online journalism, Wiley, pp. 192-208.

Pein C. (2014), “Amway journalism”, The Baffler, 28 luglio 2014, disponibile su www.thebaffler.com/blog/amway-journalism/, consultato il 20 luglio 2015.

Pérez-Peña R. (2008), “A venerable magazine energizes its web site”, The New York Times, 21 gennaio 2008, disponibile su: www.nytimes.com/2008/01/21/business/ media/21atlantic.html?_r=1, consultato il 20 luglio 2015.

Petrie N. (2011), "Have we lowered the bar on verification", Wannabe Hacks, 29 marzo 2011, disponibile su www.wannabehacks.co.uk/2011/03/29/have-we-lowe red-the-baron-verification/, consultato il 20 luglio 2015.

Pew Research Center (2015), State of the News Media 2015, disponibile su www.jo urnalism.org/files/2015/04/FINAL-STATE-OF-THE-NEWS-MEDIA1.pdf, consultato il 20 luglio 2015.

Pielke R. (2015), "Psephological pseudoscience”, The Guardian, 9 maggio 2015, disponibile su www.theguardian.com/science/political-science/2015/may/09/pseph ologicalpseudoscience, consultato il 20 luglio 2015.

Quakebot (2014), "Earthquake: 3.0 quake strikes near Brea”, Los Angeles Times, 23 ottobre 2014, disponibile su www.latimes.com/local/lanow/la-me-earthquakesae arthquake-30-quake-strikes-near-brea-calif-shcnau-story.html, consultato il 20 luglio 2015.

Rebillard F. e Smyrnaios N. (2010), "Les infomédiaires, au coeur de la filière de l'information d'actualité en ligne: Les cas de Google, Wikio et Paperblog”, Réseaux, n. 160-161, pp. 163-194.

Reckhow M. (2015), “Introducing Instant Articles”, Facebook, disponibile su http:/ /media.fb.com/2015/05/12/instantarticles/, consultato il 20 luglio 2015.

Redden J. e Witschge T. (2009), "A new news order? Online news content examined", in Fenton N., New media, old news: journalism and democracy in the digital age, Sage.

Rosen J. (2006), “The people formerly known as the audience”, in PressThink, 27 giugno 2006, disponibile su: http://archive.pressthink.org/2006/06/27/ppl_frmr. html, consultato il 20 luglio 2015.

Rushkoff D. (2003), Open source democracy: how online communication is changing offline politics, Demos.

Schudson M. (1981), Discovering the news: A social history of American newspapers, Basic Books.

Shannon C. E. (2001), "A mathematical theory of communication”, ACM SIGMOBILE Mobile Computing and Communications Review, vol. 5, n. 1, pp. 3-55.

Siapera E. (2013), "Platform infomediation and journalism”, Culture Machine, Vol. 14. 
Siapera E. (2015), “Building a safety net for European journalists”, Project report to DG Connect, 27 gennaio 2015, disponibile su: www.balcanicaucaso.org/eng/Me dia-Freedom-Net/SEEMO-interviews/Building-a-Safety-Net-for-European-Journal ists, consultato il 20 luglio 2015.

Siapera E. ed Iliadi I. (2015), “Twitter, journalism and affective labour”, Sur le journalisme/About journalism/Sobre jornalismo, vol. 4, n. 1, pp. 76-89.

Siapera E. e Spyridou L. P. (2012), "The field of online journalism: a bourdieusian analysis", The handbook of global online journalism, pp. 77-97.

Singer J. (2010), "Journalism in a network" in M. Deuze (a cura di), Managing Media Work, Sage, pp 103-109.

Smith P. (2012), "The economics of Samantha Brick and Mail Online's audiencedriven marketing machine", The Media Briefing, disponibile su www.themediabr iefing.com/article/the-economics-of-samantha-brick-mail-online-audience-driven -marketingmachine, consultato il 20 luglio 2015.

Smith J. (2015), "Here's how to get around the paywalls of The New York Times, Wall Street Journal and more: like stealing candy from a well-informed democracy", Observer, 8 gennaio 2015, disponibile su http://observer.com/2015/01/hereshow-toget-around-the-paywalls-of-the-new-york-times-wall-street-journal-and-mo re/,consultato il 20 luglio 2015.

Smythe D. W. (1981), "On the audience commodity and its work", in Durham M. G. e Kellner D. M. (a cura di) (2009), Media and cultural studies: Keyworks, Wiley, pp. 230-56.

Starkman D. (2011), "Confidence game: the limited vision of the news gurus", Columbia Journalism Review, nov.-dic. 2011, disponibile su: www.cjr.org/essay/confi dence_game.php?page=all, consultato il 20 luglio 2015.

Stevens J. (2002), "Backpack journalism is here to stay”, Online Journalism Review, n. 2.

Symes J. (2011), “The Guardian newsblog and the death of journalism”, The Louse \& the Flea, disponibile su: https://louseandflea.wordpress.com/2011/02/22/the-gu ardiannewsblog-and-the-death-of-journalism/, consultato il 20 luglio 2015.

Thompson J. B. (2005), “The new visibility”, Theory, Culture \& Society, vol. 22, n. 6, pp. 31-51.

Thurman N. e Walters A. (2013), "Live blogging-digital journalism's pivotal platform? A case study of the production, consumption, and form of live blogs at Guardian.co. uk", Digital Journalism, 1(1), 82-101.

Timmers P. (1998), "Business models for electronic markets", Electronic Markets, vol. 8, n. 2, pp. 3-8.

Ursell G. D. (2001), "Dumbing down or shaping up? New technologies, new media, new journalism”, Journalism, vol. 2, n. 2, pp. 175-196.

Van der Wurff R. (2012), “The Economics of Online Journalism”, in Siapera E. ed A. Veglis (a cura di), The Handbook of Global Online Journalism, Wiley, pp. 231250 . 
Volpe M. (2013), “10 Horrifying Display Advertising Stats”, Hubspot, 29 aprile 2013, disponibile su: http://blog.hubspot.com/marketing/horrifying-display-adve rtising-stats, consultato il 20 luglio 2015.

Vu H. T. (2013), "The online audience as gatekeeper: The influence of reader metrics on news editorial selection", Journalism, 15(8), 1094-1110.

Wardle C., Dubberley S. e Brown P. (2014), "Amateur footage: a global study of user-generated content in TV and online news output", Tow Center for Digital Journalism, a Tow/Knight report, disponibile su http://usergeneratednews. towcenter.org/wp-content/uploads/2014/05/Tow-Center-UGC-Report.pdf, consultato il 20 luglio 2015.

Warren T. (2015), “Apple News looks like Flipboard and replaces Newsstand on your iPhone or iPad", The Verge, 8 giugno 2015, disponibile su: www.theverge.c om/2015/6/8/8746289/apple-tktk-looks-like-flipboard-and-replaces-newsstandonyour-iphone, consultato il 20 luglio 2015.

Waters E., Warren C. e Dobbie M. (2006), "The changing nature of work: a global survey and case study of atypical work in the media industry", Federazione internazionale dei giornalisti, disponibile su www.ifj.org/nc/news-single-view/bac kpid/243/category/reports-publications-6/article/the-changing-nature-of-work-a-g lobal-survey-andcase-study-of-atypical-work-in-the-media-industry-a/, consultato il 20 luglio 2015.

Weiss A. S. e Joyce V. D. M. H. (2009), "Compressed dimensions in digital media occupations. Journalists in transformation”, Journalism, 10(5), 587-603. 